Pacific Journal of Mathematics

PROCESSES AND THE TWO-SIDED MARKOV PROPERTY ON
THE CIRCLE

abel KLein and LaWrence J. Landau 


\title{
PERIODIC GAUSSIAN OSTERWALDER-SCHRADER POSITIVE PROCESSES AND THE TWO-SIDED MARKOV PROPERTY ON THE CIRCLE
}

\author{
Abel Klein and Lawrence J. Landau
}

Gaussian processes in a class of stochastic processes associated with quantum systems at nonzero temperature (the periodic stochastic processes satisfying Osterwalder-Schrader (OS) positivity on the circle) are studied. A representation of the covariance function of a periodic Gaussian OS-positive process is obtained which gives a complete description of all such processes. The two-sided Markov property on the circle is studied and it is determined which periodic Gaussian OS-positive processes satisfy the two-sided Markov property on the circle. It is shown that every periodic Gaussian OS-positive process is the restriction of a periodic Gaussian two-sided Markov process. For nonperiodic Gaussian OS-positive processes it is shown that the two-sided Markov property is equivalent to the Markov property.

1. Introduction. Certain stochastic processes are associated with quantum systems (e.g., Nelson [11, 12], Simon [14], HoeghKrohn [3], Albeverio and Hoegh-Krohn [1], Klein [5, 6], Driessler, Landau and Perez [2]). If the quantum system is at a nonzero temperature $T$ then the associated stochastic process is periodic with period equal to the inverse temperature $\beta=(k T)^{-1}$, where $k$ is Boltzmann's constant (Hoegh-Krohn [3], Albeverio and HoeghKrohn [1], Driessler, Landau and Perez [2]).

The simplest example is the Ornstein-Uhlenbeck process which is associated with the quantum mechanical harmonic oscillator. The Gaussian process $X(t)$ indexed by the real line with mean zero and covariance $E(X(t) X(s))=(2 m)^{-1} \exp (-|t-s| m)$ with $m>0$ (the usual Ornstein-Uhlenbeck process) is associated with the one-dimensional harmonic oscillator with frequency $m$ (i.e., with Hamiltonian $H=$ $1 / 2\left(-d^{2} / d x^{2}+m^{2} x^{2}-m\right)$ ) at zero temperature (e.g., Simon [14]). If this harmonic oscillator is considered at a nonzero temperature $T$, then the associated stochastic process is the periodic Gaussian process $X_{\beta}(t)$ indexed by the real line with period $\beta=(k T)^{-1}$ having mean zero and covariance

$$
\begin{gathered}
E\left(X_{\beta}(t) X_{\beta}(s)\right)=(2 m[1-\exp (-m \beta)])^{-1}(\exp (-|t-s| m) \\
+\exp (-(\beta-|t-s|) m))
\end{gathered}
$$

for $|t-s| \leqq \beta$ (Hoegh-Krohn [3]). We will call $X_{\beta}(t)$ the periodic 
Ornstein-Uhlenbeck process with period $\beta$.

An important generalization of this example associates a generalized stochastic process, the free Euclidean field, with the free scalar relativistic quantum field (Nelson [12], Simon [14]). The free Euclidean field with mass $m(m>0)$ in $d+1$ space-time dimensions is the Gaussian linear stochastic process $\Phi(f, t)$ indexed by $\mathscr{S}_{r}\left(\boldsymbol{R}^{d}\right) \times \boldsymbol{R}$ (here $\mathscr{S}_{r}\left(\boldsymbol{R}^{d}\right)$ is the real vector space of real-valued infinitely differentiable functions on $\boldsymbol{R}^{d}$ which together with its derivatives decreases faster than any polynomial at infinity) with mean zero and covariance

$$
E(\Phi(f, t) \Phi(g, s))=\left(f,\left[2 \sqrt{-\Delta+m^{2}}\right]^{-1} \exp \left(-|t-s| \sqrt{-\Delta+m^{2}}\right) g\right)
$$

where $f, g \in \mathscr{S}_{r}\left(\boldsymbol{R}^{d}\right), t, s \in \boldsymbol{R}, \Delta$ is the Laplacian operator in $d$-dimensions, and $(\cdot, \cdot)$ is the $L^{2}$-inner product. This process is associated with the free scalar relativistic quantum field with mass $m$ in $d+1$ space-time dimensions at zero temperature. If the free relativistic quantum field is considered at a nonzero temperature $T$, then the stochastic process associated with it is the periodic Gaussian linear stochastic process $\Phi_{\beta}(f, t)$ indexed by $\mathscr{S}_{r}\left(\boldsymbol{R}^{d}\right) \times \boldsymbol{R}$ with period $\beta=$ $(k T)^{-1}$ having mean zero and covariance

$$
\begin{aligned}
& E\left(\Phi_{\beta}(f, t) \Phi_{\beta}(g, s)\right)=\left(f,\left[2 \sqrt{-\Delta+m^{2}}\left(1-\exp \left(-\beta \sqrt{-\Delta+m^{2}}\right)\right)\right]^{-1}\right. \\
& \left.\quad \times\left[\exp \left(-|t-s| \sqrt{-\Delta+m^{2}}\right)+\exp \left(-(\beta-|t-s|) \sqrt{-\Delta+m^{2}}\right)\right] g\right)
\end{aligned}
$$

for $f, g \in \mathscr{S}_{r}\left(\boldsymbol{R}^{d}\right), t, s \in R,|t-s| \leqq \beta$ (Hoegh-Krohn [3]).

More generally, generalized free Euclidean fields are associated with generalized free scalar relativistic quantum fields. A generalized free Euclidean field is the Gaussian linear stochastic process $\Phi(f, t)$ indexed by $\mathscr{S}_{r}\left(\boldsymbol{R}^{d}\right) \times \boldsymbol{R}$ with mean zero and covariance

$$
\begin{gathered}
E(\Phi(f, t) \Phi(g, s))=\int_{0}^{\infty} d \rho\left(m^{2}\right)\left(f,\left[2 \sqrt{-\Delta+m^{2}}\right]^{-1}\right. \\
\left.\times \exp \left(-|t-s| \sqrt{-\Delta+m^{2}}\right) g\right),
\end{gathered}
$$

where $\rho$ is a measure on $[0, \infty)$ such that $\int_{\infty}^{1} m^{-1} d \rho\left(m^{2}\right)<\infty$ (and $\int_{0}^{1} \ln m^{-1} d \rho\left(m^{2}\right)<\infty$ if $\left.d=1\right)$. This is the stochastic process associated with the quantum system at zero temperature. At a nonzero temperature $T$ we get the periodic Gaussian linear stochastic process $\Phi_{\beta}(f, t)$ indexed by $\mathscr{S}_{r}\left(\boldsymbol{R}^{d}\right) \times \boldsymbol{R}$ with period $\beta=(k T)^{-1}$ having mean zero and covariance

$$
\begin{aligned}
& E\left(\Phi_{\beta}(f, t) \Phi_{\beta}(g, s)\right)=\int_{0}^{\infty} d \rho\left(m^{2}\right)\left(f,\left[2 \sqrt{-\Delta+m^{2}}\right.\right. \\
& \left.\times\left(1-\exp \left(-\beta \sqrt{-\Delta+m^{2}}\right)\right)\right]^{-1}\left[\exp \left(-|t-s| \sqrt{-\Delta+m^{2}}\right)\right. \\
& \left.\left.+\exp \left(-(\beta-|t-s|) \sqrt{-\Delta+m^{2}}\right)\right] g\right) \text {, }
\end{aligned}
$$


for $|t-s| \leqq \beta \quad$ at $T>0$ we also need $\int_{0}^{\infty} \ln m^{-1} d \rho\left(m^{2}\right)<\infty$ if $d=2$, $\int_{0}^{1} m^{-1} d \rho\left(m^{2}\right)<\infty$ if $\left.d=1\right)$.

Other stochastic processes associated with quantum systems at nonzero temperature (including non-Gaussian processes) are discussed by Hoegh-Krohn [3], Albeverio and Hoegh-Krohn [1], and Driessler, Landau and Perez [2].

These stochastic processes associated with quantum systems have certain important properties in common. They are stationary, symmetric, and satisfy Osterwalder-Schrader (OS)-positivity, which was originally derived by Osterwalder and Schrader [13] from physical considerations in the context of quantum field theory. In the nonzero temperature case OS-positivity must be properly interpreted with respect to the circle of length $\beta$ (the precise definitions are given in $\S \S 2$ and 3 ).

In the nonperiodic case (i.e., zero temperature) OS-positive processes have been studied by Klein $[4,5,6,7,8]$.

In this article we study in detail periodic (i.e., nonzero temperature) Gaussian OS-positive processes. We obtain a representation of the covariance function of such processes which enable us to describe all periodic Gaussian OS-positive processes (Theorems 2.5, 3.3, and 3.8). In the simplest case, that of a periodic Gaussian OS-positive process $X(t)$ indexed by the real line with period $\beta(\S 2)$, we show that it has covariance function

$$
E(X(t) X(s))=\int_{0}^{\infty}\left(e^{-|t-s| a}+e^{-(\beta-|t-s|) a}\right) d \nu_{+}(a)
$$

for $|t-s| \leqq \beta$, where $\nu_{+}$is a finite measure on $[0, \infty)$ (Theorem 2.5), i.e., the most general covariance function is a positive combination of covariance functions of periodic Ornstein-Uhlenbeck processes. The general case of a periodic Gaussian OS-positive linear process indexed by $V \times \boldsymbol{R}$, where $V$ is a real vector space, is treated in $\S 3$.

For periodic processes the usual Markov property does not make sense, as the "future" winds back into the "past". The appropriate condition is the two-sided Markov property on the circle (see $\S 4)$. The periodic Ornstein-Uhlenbeck processes and the periodic free Euclidean fields are examples of processes satisfying the twosided Markov property on the circle (Corollaries 4.4 and 4.5). We determine which periodic Gaussian OS-positive processes satisfy the two-sided Markov property on the circle (Theorem 4.1). In particular we prove that the periodic Ornstein-Uhlenbeck processes are the only periodic Gaussian OS-positive processes indexed by the real line satisfying the two-sided Markov property on the circle (Corol- 
lary 4.4), and similarly for the periodic free Euclidean fields in the class of periodic generalized free Euclidean fields (Corollary 4.5).

For a periodic stationary symmetric stochastic process the twosided Markov property on the circle implies OS-positivity on the circle. In particular restrictions (see $\S 4$ for definitions) of periodic two-sided Markov processes are periodic OS-positive processes. For Gaussian processes we prove the converse, namely that every periodic Gaussian OS-positive process is the restriction of a periodic Gaussian two-sided Markov process and there exists a minimal such two-sided Markov extension (Theorem 4.6).

Finally, we look at the two-sided Markov property for (nonperiodic) Gaussian OS-positive processes ( $(5)$. In general the usual Markov property implies the two-sided Markov property, but the converse is not true. For Gaussian OS-positive processes we prove that the Markov property and the two-sided Markov property are equivalent (Corollary 5.4).

Non-Gaussian periodic OS-positive processes will be studied in a subsequent paper (Klein and Landau [9]).

2. Periodic Gaussian OS-positive processes indexed by the real line. Let $X(t)$ be a stochastic process indexed by the real line. We will call $X(t)$ a periodic stochastic process which period $\beta(\beta>0)$ if $X(t+\beta)=X(t)$ for all $t \in \boldsymbol{R}$. In this case $X(t)$ can be considered as a stochastic process indexed by the real numbers modulo $\beta$, which are identified with the circle of length $\beta$ (denoted by $S_{\beta}$ ).

The stochastic process $X(t)$ is said to be stationary if the processes $X(t)$ and $X(t+s)$ are equivalent stochastic processes for all $s \in \boldsymbol{R} ; X(t)$ is said to be symmetric if the processes $X(t)$ and $X(-t)$ are equivalent. For a periodic stochastic process with period $\beta$ considered as being indexed by the circle $S_{\beta}$, stationarity and symmetry correspond to translation and reflection on $S_{\beta}$, respectively.

A periodic stochastic process $X(t)$ with period $\beta$ is said to be OS-positive on $S_{\beta}$ in case

$$
E\left(\bar{F}\left(X\left(-t_{1}\right), \cdots, X\left(-t_{n}\right)\right) F\left(X\left(t_{1}\right), \cdots, X\left(t_{n}\right)\right)\right) \geqq 0
$$

whenever $t_{1}, \cdots, t_{n} \in[0, \beta / 2]$ and $F$ is a bounded measurable function on $\boldsymbol{R}^{n}$ (for any $n=1,2, \cdots$ ).

Let us now consider a Gaussian process $X(t)$ indexed by the real line, periodic with period $\beta$, stationary, continuous in quadratic mean, and OS-positive on $S_{\beta}$. For brevity we will call such a process a periodic Gaussian OS-positive process with period $\beta$.

A Gaussian process $X(t)$ is determined by its covariance and mean. Without loss of generality we assume its mean to be zero. If the Gaussian process is stationary, 


$$
r(t)=E(X(0) X(t))=E(X(s) X(s+t))
$$

for all $t, s \in \boldsymbol{R}$ is its covariance function.

We can characterize the covariance function of a periodic Gaussian OS-positive process.

Proposition 2.1. Let $X(t)$ be a periodic Gaussian OS-positive process with period $\beta$, and let $r(t)$ be its covariance function. Then

(i) $r(t)$ is a real-valued continuous periodic function with period $\beta$.

(ii) $r(t)$ is a positive definite function.

(iii) $r(t)$ is OS-positive on $S_{\beta}$, i.e.,

$$
\sum_{i, j=1}^{n} \bar{c}_{i} c_{j} r\left(t_{i}+t_{j}\right) \geqq 0 \text { for all } c_{1}, \cdots, c_{n} \in C, t_{1}, \cdots, t_{n} \in[0, \beta / 2] .
$$

Conversely, if $r(t)$ satisfies (i), (ii) and (iii) then it is the covariance function of a periodic Gaussian OS-positive process with period $\beta$.

Proof. Let $r(t)$ be the covariance function of a periodic Gaussian OS-positive process with period $\beta$. Then (i), (ii) are immediate. (iii) follows from the OS-positivity on $S_{\beta}$ as

$$
\left.\sum_{i, j=1}^{n} \bar{c}_{\imath} c_{j} r\left(t_{i}+t_{j}\right)=E \overline{\left(\left[\sum_{i=1}^{n} c_{i} X\left(-t_{i}\right)\right]\right.}\left[\sum_{j=1}^{n} c_{j} X\left(t_{j}\right)\right]\right) .
$$

Conversely, (i) and (ii) imply that $r(t)$ is the covariance function of a Gaussian process $X(t)$, stationary and continuous in quadratic mean. The periodicity of the process follows from the periodicity of the covariance function, as

$$
E(X(t) X(t+\beta))=r(\beta)=r(0)=E\left(X(t)^{2}\right)=E\left(X(t)^{2}\right)^{1 / 2} E\left(X(t+\beta)^{2}\right)^{1 / 2}
$$

which implies that $X(t)=X(t+\beta)$. OS-positivity on $S_{\beta}$ follows from (iii) by the same argument as in the proof of Proposition 3.1 in Klein [8], with the obvious modifications.

A function $r(t)$ satisfying (i), (ii) and (iii) will be called a periodic OS-positive covariance function with period $\beta$.

REMARK 2.2. Equivalently, a periodic OS-positive covariance function with period $\beta$ can be given as being a function $r$ on $S_{\beta}$ such that

(i' $\quad r$ is real-valued and continuous on $S_{\beta}$.

(ii') $r$ is a positive definite function on $S_{\beta}$.

(iii) $r$ is OS-positive on $S_{\beta}$. 
REMARK 2.3. Let $r(t)$ be a periodic OS-positive covariance function with period $\beta$. Then (iii) implies that $r(t) \geqq 0$ for all $t \in \boldsymbol{R}$. It also follows that $r(t)$ is symmetric (i.e., $r(t)=r(-t)$ for all $t \in \boldsymbol{R}$ ) as every real-valued positive definite function is symmetric. As a consequence, periodic Gaussian OS-positive processes are symmetric.

A symmetric function $r(t)$ satisfying (i) and (iii) of Proposition 2.1 will be called a periodic OS-positive symmetric function with period $\beta$.

The following lemma will be useful.

LEMma 2.4. Let $r(t)$ be a symmetric function. Then $r(t)$ is periodic with period $\beta$ if and only if $r(t)$ is symmetric around $\beta / 2$, i.e., $r(\beta / 2+t)=r(\beta / 2-t)$ for all $t \in \boldsymbol{R}$.

Proof. Suppose $r(t)$ is a periodic symmetric function with period B. Then

$$
r(\beta / 2+t)=r(-\beta / 2-t)=r(\beta-\beta / 2-t)=r(\beta / 2-t) .
$$

Conversely, let $r(t)$ be symmetric (around $t=0$ ) and symmetric around $\beta / 2$. Then

$$
r(\beta+t)=r(\beta / 2+(\beta / 2+t))=r(\beta / 2-(\beta / 2+t))=r(-t)=r(t) .
$$

We have already encountered periodic OS-positive functions with period $\beta$, namely the periodic functions with period $\beta$ defined by $r_{a}(t)=e^{-t a}+e^{-(\beta-t) a}$ for $t \in[0, \beta]$, with $a>0$. The positive definiteness follows from

$$
r_{a}(t)=\sum_{n \in Z} c_{n}(a) e^{i 2 \pi n t / \beta}
$$

where $c_{n}(a)=\left[(\alpha \beta)^{2}+(2 \pi n)^{2}\right]^{-1}\left[2 \beta a\left(1-e^{-\beta a}\right)\right] \geqq 0$, and $\sum_{n \in Z} c_{n}(a)=$ $1+e^{-\beta a}<\infty$ (Hoegh-Krohn [3]). The OS-positivity follows from the fact that $e^{-b t}$ is easily seen to be OS-positive for any real $b$. Up to multiplication by a constant, these are the covariance functions of periodic Ornstein-Uhlenbeck processes. Other examples are the trivial covariance functions, i.e., $r(t)$ constant. Clearly positive linear combinations of those give additional periodic OS-positive covariance functions. Remarkably, these are all.

THEOREM 2.5. Let $r(t)$ be a real-valued function of a real variable. The following are equivalent:

(i) $r(t)$ is a periodic OS-positive covariance function with period $\beta$.

(ii) $r(t)$ is a periodic OS-positive symmetric function with 
period $\beta$.

(iii) $r(t)$ is periodic with period $\beta$ and there exists a finite measure $\nu_{+}$on $[0, \infty)$ such that

$$
r(t)=\int_{0}^{\infty}\left(e^{-t a}+e^{-(\beta-t) a}\right) d \nu_{+}(a) \text { for all } t \in[0, \beta] .
$$

(iv) $r(t)$ is periodic with period $\beta$ and there exists a measure $\mu_{+}$on $[0, \infty)$ with $\int_{0}^{\infty} e^{(\beta / 2) a} d \mu_{+}(a)<\infty$ such that

$$
r(t)=\int_{0}^{\infty} \cosh [(\beta / 2-t) a] d \mu_{+}(\alpha) \text { for all } t \in[0, \beta] .
$$

Furthermore, $\nu_{+}$and $\mu_{+}$are unique.

Proof. Let us first notice that (i) $\Rightarrow$ (ii) by Remark 2.3 and that (iii) $\Rightarrow$ (iv) as $e^{-a t}+e^{-(\beta-t) a}=2 e^{-(\beta / 2) a} \cosh [(\beta / 2-t) a]$. It is also clear that (iii) $\Leftrightarrow$ (i) as $e^{-a t}+e^{-(\beta-t) a}$ is a periodic OS-positive covariance function with period $\beta$ for all $a \geqq 0$. Thus we must only prove that (ii) $\Leftrightarrow$ (iii). So let $r(t)$ be a periodic OS-positive symmetric function with period $\beta$. In particular $r(t)$ is a continuous function on $[0, \beta]$ satisfying condition (iii) of Proposition 2.1. It follows by a Theorem in Krein [10, p. 141] that there exists a finite measure $\nu$ on $\boldsymbol{R}$ with $\int_{-\infty}^{\infty} e^{-\beta a} d \nu(a)<+\infty$ such that

$$
r(t)=\int_{-\infty}^{\infty} e^{-t a} d \nu(a) \text { for all } t \in[0, \beta]
$$

Such a measure $\nu$ is unique due to the uniqueness of the Fourier transform of a finite measure on $\boldsymbol{R}$ and to an analytic continuation argument. By Lemma $2.4 r(t)$ is symmetric around $\beta / 2$. It follows that for all $t \in[0, \beta / 2] r(\beta / 2+t)=r(\beta / 2-t)$ and thus

$$
\int_{-\infty}^{\infty} e^{-t a} e^{-(\beta / 2) a} d \nu(a)=\int_{-\infty}^{\infty} e^{t a} e^{-(\beta / 2) a} d \nu(a)=\int_{-\infty}^{\infty} e^{-t a} e^{(\beta / 2) a} d \nu(-a)
$$

for all $t \in[0, \beta / 2]$. By the same argument used to prove the uniqueness of $\nu$ it now follows that $e^{-(\beta / 2) a} d \nu(a)=e^{(\beta / 2) a} d \nu(-a)$ and thus that $d \nu(-a)=e^{-\beta a} d \nu(a)$. Hence there exists a unique finite measure $\nu_{+}$with support on $[0, \infty)$ such that

$$
d \nu(a)=d \nu_{+}(a)+e^{\beta a} d \nu_{+}(-a) .
$$

Therefore

$$
r(t)=\int_{0}^{\infty}\left(e^{-t a}+e^{-(\beta-t) a}\right) d \nu_{+}(a) \text { for all } t \in[0, \beta]
$$


for a unique measure $\nu_{+}$on $[0, \infty]$.

Combining Proposition 2.1 and Theorem 2.5 we get:

CoRollary 2.6. Let $X(t)$ be a Gaussian process indexed by the real line. Then $X(t)$ is a periodic Gaussian OS-positive process with period $\beta$ if and only if there exists a finite measure $\nu_{+}$on $[0, \infty)$ such that

$$
E(X(t) X(s))=\int_{0}^{\infty}\left(e^{-\tau a}+e^{-(\beta-\tau) a}\right) d \nu_{+}(a)
$$

for all $t, s \in R$ with $\tau \in[0, \beta], \tau \equiv t-s \bmod \beta$.

3. Periodic Gaussian OS-positive processes indexed by $V \times \boldsymbol{R}$. Let us now consider a stochastic process $\Phi(v, t)$ indexed by $V \times \boldsymbol{R}$, where $V$ is a real vector space, which is linear (i.e., $\Phi(v, t)$ is linear in $v$ for each $t \in \boldsymbol{R})$. Such a process will be said to be periodic with period $\beta(\beta>0)$ if $\Phi(v, t+\beta)=\Phi(v, t)$ for all $v \in \boldsymbol{R}$. In this case $\Phi(v, t)$ can be considered to be a stochastic process indexed by $V \times S_{\beta}$.

We will call the stochastic process $\Phi(v, t)$ stationary if the processes $\Phi(v, t)$ and $\Phi(v, t+s)$ are equivalent for all $s \in \boldsymbol{R} ; \Phi(v, t)$ will be said to be symmetric if the processes $\Phi(v, t)$ and $\Phi(v,-t)$ are equivalent. If $\Phi(v, t)$ is a periodic stochastic process with period $\beta$, stationary and symmetry correspond to translation and reflection on the circle $S_{\beta}$, respectively.

A periodic stochastic process $\Phi(v, t)$ with period $\beta$ is said to be OS-positive on $S_{\beta}$ if

$$
E\left(\bar{F}\left(\Phi\left(v_{1},-t_{1}\right), \cdots, \Phi\left(v_{n},-t_{n}\right)\right) F\left(\Phi\left(v_{1}, t_{1}\right), \cdots, \Phi\left(v_{n}, t_{n}\right)\right)\right) \geqq 0
$$

whenever $t_{1}, \cdots, t_{n} \in[0, \beta / 2], v_{1} \cdots, v_{n} \in V$, and $F$ is a bounded measurable function on $\boldsymbol{R}^{n}$ (for any $n=1, \cdots$ ).

Let us now consider a Gaussian linear stochastic procss indexed by $V \times \boldsymbol{R}$, periodic with period $\beta$, stationary, continuous in quadratic mean (i.e., for each $v \in V$ the process $\Phi(v, t)$ indexed by the real line is continuous in quadratic mean), and OS-positive on $S_{\beta}$. Such a process will be called a periodic Gaussian OS-positive process with period $\beta$.

A Gaussian process is determined by its covariance and mean. Without loss of generality we may assume the mean to be zero. If the process is stationary we can assume, also without loss of generality, that $E\left(\Phi(v, 0)^{2}\right) \neq 0$ for all $v \in V$ (i.e., $\Phi(v, 0)$ is not the zero random variable; by stationarity neither are $\Phi(v, t)$ for any $t \in R)$. 
Thus $V$ can be given the inner product $\langle v, w\rangle=E(\Phi(v, 0) \Phi(w, 0))$ and the $\operatorname{map} v \mapsto \Phi(v, 0)$ extends to the completion $\bar{V}$ of $V$ under this inner product. It follows that we could assume without loss of generality that $V$ is a real Hilbert space with $\langle v, w\rangle=E(\Phi(v, 0) \Phi(w, 0))$. For reasons that will be clear later we will only assume that $V$ is a real Hilbert space with inner product $\langle\cdot, \cdot\rangle$ and that there exists a finite constant $C$ such that $E\left(\Phi(v, 0)^{2}\right) \leqq C\|v\|^{2}$ for all $v \in V$. We will denote by $V_{c}$ the complex Hilbert space that is the complexification of $V$ and we will extend by linearity the process $\Phi(v, t)$ to a complex process indexed by $V_{c} \times \boldsymbol{R}$, which we will also denote by $\Phi(v, t)$. By $\mathscr{L}\left(V_{c}\right), \mathscr{L}(V)$ we will denote the Banach spaces of bounded linear operators on $V_{c}, V$, respectively.

Let $\Phi(v, t)$ be a stationary Gaussian linear stochastic process. As

$$
\begin{aligned}
& |E(\Phi(v, 0) \Phi(w, t))| \leqq E\left(|\Phi(v, 0)|^{2}\right)^{1 / 2} E\left(|\Phi(w, t)|^{2}\right)^{1 / 2} \\
& \quad=E\left(|\Phi(v, 0)|^{2}\right)^{1 / 2} E\left(|\Phi(w, 0)|^{2}\right)^{1 / 2} \leqq C\|v\|\|w\|
\end{aligned}
$$

for $v, w \in V_{c}, t \in \boldsymbol{R}$, we can define an operator-valued function $r: \boldsymbol{R} \rightarrow \mathscr{L}\left(V_{c}\right)$ by $\left.\langle v, r(t) w\rangle=E(\overline{\Phi(v, 0}) \Phi(w, t)\right)$ for all $v, w \in V_{c}, t \in \boldsymbol{R}$. For each $t \in \boldsymbol{R} r(t)$ is a well defined bounded linear operator on $V_{c}$ with $\|r(t)\| \leqq C$, leaving $V$ invariant. We will call $r(t)$ the (operatorvalued) covariance function of the process $\Phi(v, t)$.

Proposition 3.1. Let $\Phi(v, t)$ be a periodic Gaussian OS-positive process with period $\beta$ indexed by $V \times \boldsymbol{R}$, and let $r(t)$ be its (operatorvalued) covariance function. Then

(i) $r(t)$ leaves $V$ invariant, is weakly continuous, and periodic with period $\beta$.

(ii) $r(t)$ is an operator-valued positive definite function, i.e.,

$\sum_{i, j=1}^{n}\left\langle v_{i}, r\left(t_{j}-t_{i}\right) v_{j}\right\rangle \geqq 0$ for all $v_{1}, \cdots, v_{n} \in V_{c}, t_{1}, \cdots, t_{n} \in \boldsymbol{R}$.

(iii) $r(t)$ is OS-positive on $S_{\beta}$, i.e.,

$$
\sum_{i, j=1}^{n}\left\langle v_{i}, r\left(t_{i}+t_{j}\right) v_{j}\right\rangle \geqq 0 \quad \text { for all } v_{1}, \cdots, v_{n} \in V_{c}, t_{1}, \cdots, t_{n} \in[0, \beta / 2] .
$$

Conversely, if $r: \boldsymbol{R} \rightarrow \mathscr{L}\left(V_{c}\right)$ satisfies (i), (ii) and (iii) then it is the covariance function of a periodic Gaussian OS-positive process with period $\beta$ indexed by $V \times \boldsymbol{R}$.

Proof. Similar to the proof of Proposition 2.1. Just notice that 


$$
\sum_{i, j=1}^{n}\left\langle v_{i}, r\left(t_{i}+t_{j}\right) v_{j}\right\rangle=E\left(\overline{\left[\sum_{i=1}^{n} \Phi\left(v_{i},-t_{i}\right)\right]}\left[\sum_{j=1}^{n} \Phi\left(v_{j}, t_{j}\right)\right]\right) .
$$

A function $r: \boldsymbol{R} \rightarrow \mathscr{L}\left(V_{c}\right)$ satisfying (i), (ii) and (iii) will be called a periodic OS-positive covariance function with period $\beta$. In view of (i) we can also consider $r$ as a function $r: \boldsymbol{R} \rightarrow \mathscr{L}(V)$ whose extension to $V_{c}$ satisfies (i), (ii) and (iii).

REMARK 3.2. Remarks 2.2 and 2.3 apply to operator-valued covariance functions with the obvious modifications. In particular $r(t)$ is symmetric (i.e., $r(t)=r(-t)$ for all $t \in \boldsymbol{R}$ ), and a bounded nonnegative self-adjoint operator leaving $V$ invariant for all $t \in \boldsymbol{R}$. It will follow from the next theorem that (i), (iii), and symmetry imply (ii).

We will now obtain a representation for periodic OS-positive operator-valued covariance functions which extends the representation obtained in Theorem 2.5 for real-valued covariance functions. For that we need operator-valued measures. An operator-valued measure on an interval $I \subset \boldsymbol{R}$ with values in $\mathscr{L}(V)$ (or equivalently, with values on $\mathscr{L}\left(V_{c}\right)$ leaving $V$ invariant) is an additive map $\Gamma$ from the Borel sets in $I$ to the bounded nonnegative self-adjoint operators on $V_{c}$ leading $V$ invariant, such that if $\left\{A_{n}\right\}$ in an increasing sequence of Borel subsets of $I$ and $A=\bigcup_{n=1}^{\infty} A_{n}$, then $\Gamma\left(A_{n}\right) \rightarrow \Gamma(A)$ weakly as $n \rightarrow \infty$. Given such an $\mathscr{L}(V)$-valued measure $\Gamma$ and $v \in V_{c}, \nu_{v}(A)=\langle v, \Gamma(A) v\rangle$ for $A$ a Borel subset of $I$ gives a finite measure on $I$, and by polarization $\nu_{v, w}(A)=\langle v, \Gamma(A) w\rangle$ gives a complex measure $\nu_{v, w}$ on $I$ for all $v, w \in V_{c}$. Moreover the map $(v, w) \mapsto \nu_{v, w}$ is sesquilinear. If $f$ is a bounded measurable function or $I$, by $T=\int_{I} f(a) d \Gamma(a)$ (weakly) we mean that $\langle v, T w\rangle=$ $\int_{I} f(a) d \nu_{v, w}(a)$ for all $v, w \in V_{c}$. To show that $T$ is well defined let us first assume that $f$ is nonnegative. Then $(v, w) \mapsto \int_{I} f(a) d \nu_{v, w}(a)$ is a positive semi-definite sesquilinear form on $V_{c}$, so by the CauchySchwarz inequality

$$
\begin{aligned}
\left|\int_{I} f(a) d \nu_{v, w}(a)\right| & \leqq\left(\int_{I} f(a) d \nu_{v}(a)\right)^{1 / 2}\left(\int_{I} f(a) d \nu_{w}(a)\right)^{1 / 2} \\
& \leqq\|f\|_{\infty}\|\Gamma(I)\|\|v\|\|w\|
\end{aligned}
$$

It follows that $\int_{I} f(a) d \Gamma(a)$ is a well defined bounded operator on $V_{c}$ leaving $V$ invariant. For an arbitrary bounded measurable function $f$ or $I, \int_{I} f(a) d \Gamma(a)$ is defined by writing $f$ as a linear combination of four bounded nonnegative measurable functions. For unbounded measurable functions $f$ the same analysis applies as long as one has a 
bound of the form $\int_{I}|f(a)| d \nu_{v}(a) \leqq D\|v\|^{2}$ for all $v \in V$ for some finite constant $D$. We will denote by $L^{2}\left(I, V_{c} ; d \Gamma\right)$ the complex Hilbert space which is the completion of the space of bounded measurable functions $F: I \rightarrow V_{c}$ with finite-dimensional range and inner product $(F, G)=\int\langle F(a), d \Gamma(a) G(a)\rangle$, i.e., if $F(a)=\sum_{i=1}^{n} f_{i}(a) v_{i}, G(a)=\sum_{i=1}^{n} g_{i}(a) v_{i}$, where $f_{i}, g_{i}, i=1, \cdots, n$, are bounded measurable functions on $I$, $v_{1}, \cdots, v_{n} \in V_{c}$ (any $F, G$ with finite-dimensional range can be written in this form), then

$$
(F, G)=\sum_{i, j=1}^{n} \int_{I} \bar{f}_{i}(a) g_{j}(a) d \nu_{v_{i}, v_{j}}(a) .
$$

The positive semi-definiteness is proven by an approximation argument using characteristic functions. By $L^{2}(I, V ; d \Gamma)$ we denote the real Hilbert space of $V$-valued functions in $L^{2}\left(I, V_{c} ; d \Gamma\right)$.

THEOREM 3.3. A function $r: \boldsymbol{R} \rightarrow \mathscr{L}\left(V_{c}\right)$ is a periodic OS-positive covariance function with period $\beta$ if and only if $r(t)$ is periodic with period $\beta$ and there exists an operator-valued measure $\Gamma_{+}$ on $[0, \infty)$ with values in $\mathscr{L}(V)$ such that

$$
r(t)=\int_{0}^{\infty}\left(e^{-t a}+e^{-(\beta-t) a}\right) d \Gamma_{+}(a) \quad(w e a k l y) \text { for all } t \in[0, \beta] .
$$

Moreover such $\Gamma_{+}$is unique.

Proof. Let us first prove that if $\Gamma_{+}$is an operator-valued measure or $[0, \infty)$ with values in $\mathscr{L}(V)$ and if $r: \boldsymbol{R} \mapsto \mathscr{L}\left(V_{c}\right)$ is a periodic function with period $\beta$ defined by $r(t)=\int_{0}^{\infty}\left(e^{-t a}+e^{-(\beta-t) a}\right) d \Gamma_{+}(\alpha)$ (weakly) for $t \in[0, \beta)$, then $r(t)$ is a periodic OS-positive covariance function. Condition (i) is immediate, we must only prove (ii) and (iii). Those will follow from the fact that $e^{-t a}+e^{-(\beta-t) a}$ is a realvalued OS-positive covariance function for $a \geqq 0$. We will show first that $r(t)$ is OS-positive on $S_{\beta}$. So let $v_{1}, \cdots, v_{n} \in V, t_{1}, \cdots, t_{n} \in$ $[0, \beta / 2]$. Then

$$
\begin{aligned}
\sum_{i, j=1}^{n}\left\langle v_{i}, r\left(t_{i}+t_{j}\right) v_{j}\right\rangle=\sum_{i, j=1}^{n} \int_{0}^{\infty}\left(e^{-\left(t_{i}+t_{j}\right) a}+e^{-\left(\beta-\left(t_{\imath}+t_{j}\right) a a\right.}\right) d \nu_{v_{i}, v_{j}}(a) \\
=\sum_{i, j=1}^{n}\left(e^{-t_{i} a} v_{\imath}, e^{-t_{j} a} v_{j}\right)+\sum_{i, j=1}^{n}\left(e^{-\left(\beta / 2-t_{\imath}\right) a} v_{i}, e^{-\left(\beta / 2-t_{j}\right) a} v_{j}\right) \\
=\left(\sum_{i=1}^{n} e^{-t_{i} a} v_{i}, \sum_{j=1}^{n} e^{-t_{j} a} v_{j}\right)+\left(\sum_{j=1}^{n} e^{-\left(\beta / 2-t_{\imath}\right) a} v_{i}, \sum_{i=1}^{n} e^{-\left(\beta / 2-t_{j}\right) a} v_{j}\right) \geqq 0 .
\end{aligned}
$$

Here $(\cdot, \cdot)$ is the inner product in $L^{2}\left([0, \infty), V_{c}, d \Gamma_{+}\right)$, and $\nu_{v, w}(\cdot)=$ $\left\langle v, \Gamma_{+}(\cdot) w\right\rangle$. 
To prove that $r(t)$ is positive definite, let us recall that

$$
e^{-t a}+e^{-(\beta-t) a}=\sum_{n \in Z} c_{n}(a) e^{i 2 \pi n t / \beta} \text { for all } t \in[0, \beta],
$$

where

$$
c_{n}(a)=\left[(a \beta)^{2}+(2 \pi n)^{2}\right]^{-1}\left[2 \beta a\left(1-e^{-\beta a}\right)\right] \geqq 0,
$$

so

$$
\sum_{n \in Z} c_{n}(a)=1+e^{-\beta a}<+\infty \quad \text { (Hoegh-Krohn [3]), }
$$

and the right-hand side (for all $t \in R$ ) defines a periodic function with period $\beta$ on $\boldsymbol{R}$. Thus for all $t \in \boldsymbol{R}$

$$
r(t)=\int_{0}^{\infty}\left(\sum_{n \in \boldsymbol{Z}} c_{n}(a) e^{i 2 \pi n t / \beta}\right) d \Gamma_{+}(\alpha)=\sum_{n \in \boldsymbol{Z}} e^{i 2 \pi n t / \beta} \int_{0}^{\infty} c_{n}(a) d \Gamma_{+}(\alpha) .
$$

The interchange of summation and integration can be justified by looking at $\langle v, r(t) v\rangle$ and using Fubini's theorem. Hence, if $v_{1}, \cdots$, $v_{n} \in V_{c}, t_{1}, \cdots, t_{n} \in \boldsymbol{R}$,

$$
\sum_{i, j=1}^{n}\left\langle v_{i}, r\left(t_{j}-t_{i}\right) v_{j}\right\rangle=\sum_{n \in Z}\left\langle\sum_{j=1}^{n} e^{i 2 \pi n t_{i} / \beta} v_{i}, \int_{0}^{\infty} c_{n}(a) d \Gamma_{+}(a) \sum_{j=1} e^{i 2 \pi n t_{j} / \beta} v_{j}\right\rangle \geqq 0
$$

as $c_{n}(a) \geqq 0$ for all $n \in Z, a \in[0, \infty)$. Thus $\int_{0}^{\infty}\left(e^{-t a}+e^{-(\beta-t) a}\right) d \Gamma_{+}(a)$ is a periodic OS-positive covariance function with period $\beta$.

Now let $r: \boldsymbol{R} \rightarrow \mathscr{L}\left(V_{c}\right)$ be a periodic OS-positive covariance function with period $\beta$. For each $v \in V_{c}\langle v, r(t) v\rangle$ is a real-valued periodic OS-positive function with period $\beta$, so by Theorem 2.5 there exists a unique finite measure $\nu_{v}$ on $[0, \infty)$ such that $\langle v$, $r(t) v\rangle=\int_{0}^{\infty}\left(e^{-t a}+e^{-(\beta-t) a}\right) d \nu_{v}(a)$ for all $t \in[0, \beta]$. It follows by polarization that for every $v, w \in V_{c}$ there exists a complex measure $\nu_{v, w}$ on $[0, \infty)$, real-valued if $v, w \in V$, such that $\langle v, r(t) w\rangle=\int_{0}^{\infty}\left(e^{-t a}+\right.$ $\left.e^{-(\beta-t) a}\right) d \nu_{v, w}(a)$, and moreover $(v, w) \mapsto \nu_{v, w}$ is a sesquilinear map on $V_{c}$. Thus if $A$ is a Borel subset of $[0, \infty)$ then $(v, w) \mapsto \nu_{v, w}(A)$ is a positive semi-definite sesquilinear form on $V_{c}$, real-valued on $V$. Hence by the Cauchy-Schwarz inequality

$$
\begin{gathered}
\left|\nu_{v, w}(A)\right| \leqq \nu_{v}(A)^{1 / 2} \nu_{w}(A)^{1 / 2} \leqq\left(\int_{0}^{\infty} d \nu_{v}(a)\right)^{1 / 2}\left(\int_{0}^{\infty} d \nu_{w}(a)\right)^{1 / 2} \\
=\langle v, r(0) v\rangle^{1 / 2}\langle w, r(0) w\rangle^{1 / 2} \leqq\|r(0)\|\|v\|\|w\| \leqq C\|v\|\|w\| \\
\text { for all } v, w \in V_{c} .
\end{gathered}
$$

Thus there exists a unique bounded nonnegative self-adjoint operator $\Gamma_{+}(A)$ on $V_{c}$ leaving $V$ invariant such that $\nu_{v, w}(A)=\left\langle v, \Gamma_{+}(A) w\right\rangle$ 
for all $v, w \in V_{c}$. The map $A \mapsto \Gamma_{+}(A)$ is clearly an operator-valued measure on $[0, \infty)$ with values in $\mathscr{L}(V)$. Moreover, for all $v, w \in V_{c}$ $\left\langle v, \int_{0}^{\infty}\left(e^{-t a}+e^{-(\beta-t) a}\right) d \Gamma_{+}(a) w\right\rangle=\int_{0}^{\infty}\left(e^{-t a}+e^{-(\beta-t) a}\right) d \nu_{v, w}(a)=\langle v, r(t) w\rangle$ for $t \in[0, \beta]$. Therefore $r(t)=\int_{0}^{\infty}\left(e^{-t a}+e^{-(\beta-t) a}\right) d \Gamma_{+}(a)$ (weakly) for all $t \in[0, \beta]$.

The uniqueness of $\Gamma_{+}$follows from the uniqueness of $\nu_{v}$ for each $v \in V_{c}$.

REMARK 3.4. Equivalently, a periodic OS-positive covariance function $r(t)$ with period $\beta$ is given by $r(t)=\int_{-\infty}^{\infty} e^{-t a} d \Gamma(a)$ for $t \epsilon$ $[0, \beta]$ for an operator-valued measure $\Gamma$ on $R$ with values in $\mathscr{L}(V)$ such that $d \Gamma(a)=d \Gamma_{+}(a)+e^{\beta a} d \Gamma_{+}(-a)$, where $\Gamma_{+}$is an operatorvalued measure with support on $[0, \infty)$ and values in $\mathscr{L}(V)$.

REMARK 3.5. Let $\Phi_{\beta_{0}}(v, t)$ be a periodic Gaussian OS-positive process with period $\beta_{0}$ indexed by $V_{0} \times \boldsymbol{R}$, where $V_{0}$ is a real vector space. It follows from Theorem 3.3 and its proof (see also Theorem 3.8) that there exists a unique inner product $(\cdot, \cdot)$ on $V_{0}$ and an unique operator-valued measure $\Gamma_{+}$on $[0, \infty)$ with values in $\mathscr{L}(V)$, where $V$ is the completion of $V_{0}$ in the inner product $(\cdot, \cdot)$, such that $(v, w)=\int_{0}^{\infty}\left(v, d \Gamma_{+}(a) w\right)$ for all $v, w \in V$, and if $r_{\beta_{0}}(t)$ is the covariance function of the periodic Gaussian OS-positive process $\Phi_{\beta_{0}}(v, t)$ (defined with respect to the inner product $(\cdot, \cdot)$ ) then

$$
r_{\beta_{0}}(t)=\int_{0}^{\infty}\left(e^{-t a}+e^{-\left(\beta_{0}-t\right) a}\right) d \Gamma_{+}(a) \text { for } t \in[0, \beta] .
$$

Moreover, if we define the periodic function with period $\beta r_{\beta}: \boldsymbol{R} \rightarrow$ $\mathscr{L}(V)$ by $r_{\beta}(t)=\int_{0}^{\infty}\left(e^{-t a}+e^{-(\beta-t) a}\right) d \Gamma_{+}(a)$ for $t \in[0, \beta]$, then $r_{\beta}(t)$ is a periodic OS-positive covariance function with period $\beta$ for any $\beta>0$.

Thus, starting from a given periodic Gaussian OS-positive process $\Phi_{\beta_{0}}(v, t)$ with period $\beta_{0}$, indexed by $V_{0} \times \boldsymbol{R}$, we can construct a periodic Gaussian OS-positive process $\Phi_{\beta}(v, t)$ indexed by $V_{0} \times \boldsymbol{R}$ with period $\beta$ for any $\beta>0$. Knowledge of one of the $\Phi_{\beta}(v, t)$ is equivalent to knowledge of all.

REMARK 3.6. As $\beta \rightarrow \infty, r_{\beta}(t) \rightarrow r_{\infty}(t)$ weakly for all $t \in \boldsymbol{R}$, where

$$
r_{\infty}(t)=\int_{0}^{\infty} e^{-|t| a} d \Gamma_{+}(a)+\Gamma_{+}(\{0\})
$$

is a (nonperiodic) OS-positive covariance function from which we 
can construct a (nonperiodic) Gaussian OS-positive process $\Phi_{\infty}(v, t)$ (Klein [8]). In case $\Gamma_{+}(\{0\}) \neq 0$ this process is different from the (nonperiodic) Gaussian OS-positive process naturally associated with $\Gamma_{+}$, namely the one with covariance function $r(t)=\int_{0}^{\infty} e^{-|t| a} d \Gamma_{+}(a)$ (see Klein [8, Remark 2.7]). Of course $r_{\infty}(t)$ can be rewritten as $r_{\infty}(t)=\int_{0}^{\infty} e^{-|t| a} d \Gamma_{+}^{\prime}(a)$, where $\Gamma_{+}^{\prime}=\Gamma_{+}$on $(0, \infty)$ and $\Gamma_{+}^{\prime}(\{0\})=2 \Gamma_{+}(\{0\})$, and vice-versa (see also Theorems 5.1 and 5.2).

REMARK 3.7. One can also obtain periodic Gaussian OS-positive process with any period $\beta>0$ from $\Phi_{\beta_{0}}(v, t)$ by scaling. These processes are different from the ones we discussed in Remark 3.5 and are not very interesting. The scaled covariance functions are $\widetilde{r}_{\beta}(t)=$ $r_{\beta_{0}}\left(\left(\beta_{0} / \beta\right) t\right)$, and thus

$$
\widetilde{r}_{\beta}(t)=\int_{0}^{\infty}\left(e^{-t a}+e^{-(\beta-t) a}\right) d \Gamma_{+}\left(\left(\beta / \beta_{0}\right) a\right)
$$

corresponds to a scaling of $\Gamma_{+} . \quad$ As $\beta \rightarrow \infty \quad \widetilde{r}_{\beta}(t) \rightarrow \int_{0}^{\infty}\left(1+e^{-\beta_{0} a}\right) d \Gamma_{+}(a)$ (weakly), which is a trivial covariance function.

Theorem 3.3 can be reformulated in terms of a semigroup in a bigger Hilbert space, on the lines of Theorem 2.3 in Klein [8].

THEOREM 3.8. Let $V$ be a real Hilbert space. Then $r: \boldsymbol{R} \rightarrow$ $\mathscr{L}(V)$ is a periodic Gaussian OS-positive covariance function with period $\beta$ if and only if $r(t)$ is periodic with period $\beta$ and there exists a real Hilbert space $W$, a nonnegative self-adjoint operator $H$ on $W$, and a continuous linear map $j: V \rightarrow W$ such that

$$
r(t)=j^{*}\left(e^{-t H}+e^{-(\beta-t) H}\right) j \text { for } t \in[0, \beta] .
$$

Moreover, $W, H$, and $j$ are uniquely determined (up to isomorphisms) by the requirement that the linear span of $\left\{e^{-t H} j v \mid t \geqq 0\right.$, $v \in V\}$ be dense in $W$.

Proof. Let $r(t)$ be a periodic Gaussian OS-positive covariance function with period $\beta$. By Theorem 3.3 there exists an operatorvalued measure $\Gamma_{+}$on $[0, \infty)$ with values in $\mathscr{L}(V)$ such that $r(t)=$ $\int_{0}^{\infty}\left(e^{-t a}+e^{-(\beta-t) a}\right) d \Gamma_{+}(a)$ for $t \in[0, \beta]$. So let $W=L^{2}\left([0, \infty), V ; d \Gamma_{+}\right)$ and $j: V \rightarrow W$ be given by $(j v)(a)=v$ for all $a \in[0, \infty)$. Then $j$ is a continuous linear map, as

$$
\begin{aligned}
(j v, j v) & =\int_{0}^{\infty}\left\langle v, d \Gamma_{+}(a) v\right\rangle \leqq \int_{0}^{\infty}\left\langle v,\left(1+e^{-\beta a}\right) d \Gamma_{+}(a) v\right\rangle \\
& =\langle v, r(0) v\rangle \leqq\|r(0)\|\langle v, v\rangle \quad \text { so } \quad\|j\| \leqq\|r(0)\|^{1 / 2}
\end{aligned}
$$


We now define $H$ as the negative of the infinitesimal generator of the strongly continuous self-adjoint semigroup $P(t)$ on $W$ given by $(P(t) F)(a)=e^{-t a} F(a)$ for all $F \in W$ and $t \geqq 0$. $H$ is thus a nonnegative self-adjoint operator on $W$ with $P(t)=e^{-t H}$. Moreover,

$$
\begin{aligned}
\langle v, r(t) w\rangle & =\int_{0}^{\infty}\left(e^{-t a}+e^{-(\beta-t) a}\right)\left\langle v, d \Gamma_{+}(a) w\right\rangle \\
& =\left(j v,\left(e^{-t H}+e^{-(\beta-t) H}\right) j w\right) \text { for all } v, w \in V, t \in[0, \beta],
\end{aligned}
$$

and hence $r(t)=j^{*}\left(e^{-t H}+e^{-(\beta-t) H}\right) j$ for all $t \in[0, \beta]$. It is also true that the linear span of $\left\{e^{-t a} j v \mid t \geqq 0, v \in V\right\}$ is dense in $L^{2}([0, \beta), V$; $\left.d \Gamma_{+}\right)$.

Conversely, let $W$ be a real Hilbert space, $j: V \rightarrow W$ a continuous linear map, and $H$ a nonnegative self-adjoint operator on $W$, and let us define $r: \boldsymbol{R} \rightarrow \mathscr{L}(V)$ as the periodic function with period $\beta$ such that $r(t)=j^{*}\left(e^{-t H}+e^{-(\beta-t) H}\right) j$ for $t \in[0, \beta]$. We let $E$ be the spectral projection of $H$ so $e^{-t H}=\int_{0}^{\infty} e^{-t a} d E(a)$ for all $t \geqq 0$. In par-

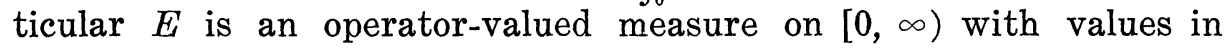
$\mathscr{L}(W)$; we let $\Gamma_{+}(\cdot)=j^{*} E(\cdot) j$. Then $\Gamma_{+}$is an operator-valued measure on $[0, \infty)$ with values in $\mathscr{L}(V)$ and

$$
\begin{aligned}
r(t) & =j^{*}\left(e^{-t H}+e^{-(\beta-t) H}\right) j=j^{*}\left(\int_{0}^{\infty}\left(e^{-t a}+e^{-(\beta-t) a}\right) d E(a)\right) j \\
& =\int_{0}^{\infty}\left(e^{-t a}+e^{-(\beta-t) a}\right) d \Gamma_{+}(a) \text { for } t \in[0, \beta] .
\end{aligned}
$$

It follows from Theorem 3.3 that $r(t)$ is a periodic OS-positive covariance function with period $\beta$.

The uniqueness of $W, H$, and $j$ with the requirement of the linear span of $\left\{e^{-t H} j v \mid t \geqq 0, v \in V\right\}$ being dense in $W$ follows from

$$
\begin{aligned}
\left(e^{-t H} j v, e^{-s H} j w\right) & =\int_{0}^{\infty} e^{-(t+s) a}(j v, d E(a) j w) \\
& =\int_{0}^{\infty} e^{-(t+s) a}\left\langle v, d \Gamma_{+}(a) w\right\rangle \text { for all } s, t \geqq 0, v, w \in V,
\end{aligned}
$$

and from the uniqueness of $\Gamma_{+}$given by Theorem 3.3.

REMARK 3.9. The analysis in Remarks 3.5 and 3.6 can be reformulated in terms of Theorem 3.8 which gives a prescription for constructing periodic Gaussian OS-positive processes $\Phi_{\beta}(v, t)$ indexed by $V_{0} \times \boldsymbol{R}\left(V_{0}\right.$ a real vector space) for any period $\beta>0$. First, one furnishes $V_{0}$ with an inner product and completes $V_{0}$ into a real Hilbert space $V$. Second, one picks a real Hilbert space $W$ having $V$ as a subspace. Third, one chooses a nonnegative self-adjoint operator $H$ on $W$. Then the periodic function $r_{\beta}: R \rightarrow \mathscr{L}(V)$ with 
period $\beta$ given by $r_{\beta}(t)=q\left(e^{-t H}+e^{-(\beta-t) H}\right) q^{*}$ for $t \in[0, \beta]$, where $q$ is the orthogonal projection of $W$ onto $V$, is a periodic OS-positive covariance function with period $\beta$ for any $\beta>0$. All periodic Gaussian OS-positive processes are obtained in this way. Moreover $r_{\infty}(t)=q\left(e^{-|t| H}+E(\{0\})\right) q^{*}$ for all $t \in \boldsymbol{R}$. The natural (nonperiodic) OS-positive function associated with $W$ and $H$ would be $r(t)=$ $q e^{-|t| H} q^{*}$ (Klein [8, Theorem 2.3]). If $H$ does not have zero as an eigenvalue we have $r_{\infty}(t)=r(t)$ (see also Theorems 5.1 and 5.2).

REMARK 3.10. We may, without loss of generality, suppose that $H$ does not have zero as an eigenvalue, since if it does, the process may be decomposed into stationary periodic Gaussian OS-positive processes with nonzero mean and such that $H$ does not have zero as an eigenvalue. Let $X(t)$ be a Gaussian process indexed by $\boldsymbol{R}$ with mean $m$ and covariance $\widehat{C}\left(t_{1}, t_{2}\right)$. The characteristic function is

$$
E_{m}\left(e^{i X(f)}\right)=e^{i m \int d t f(t)} e^{-1 / 2 \oint d t_{1} d t_{2} f\left(t_{1}\right) f\left(t_{2}\right) \hat{C}\left(t_{1}, t_{2}\right)}
$$

where $X(f)=\int d t f(t) X(t)$. Now consider the process $X(t)$ with characteristic function

$$
E\left(e^{i X(f)}\right)=\sqrt{\frac{b}{2 \pi}} \int_{-\infty}^{\infty} d m e^{-(1 / 2 b) m^{2}} E_{m}\left(e^{i X(f)}\right) .
$$

That is, we have decomposed the expectation $E$ as an integral over the expectations $E_{m}$. Then $X(t)$ is a Gaussian process with mean zero and covariance $C\left(t_{1}, t_{2}\right)=\hat{C}\left(t_{1}, t_{2}\right)+b$. If now $X(t)$ is a stationary periodic process with covariance $r(t)=\int_{0}^{\infty}\left(e^{-t a}+e^{-(\beta-t) a}\right) d \nu_{+}(a)$ for $t \in[0, \beta]$, we write

$$
\begin{aligned}
& r(t)=b+\hat{r}(t), \text { where } b=2 \nu_{+}(0), \\
& \widehat{r}(t)=\int_{0}^{\infty}\left(e^{-t a}+e^{-(\beta-t) a}\right) d \nu_{+}(a) \text { for } t \in[0, \beta],
\end{aligned}
$$

and $\nu_{+}=(b / 2) \delta(0)+\hat{\nu}_{+}\left(\hat{\nu}_{+}\right.$has no support at $\left.a=0\right)$. The previous discussion shows that the process can be decomposed into processes with mean $m$ and covariance $\hat{r}(t)$.

A similar discussion can be given for the Gaussian process $\Phi(v, t)$ indexed by $V \times \boldsymbol{R}$. For example, if $l_{1}, \cdots l_{n}$ are linear functionals on $V$, and $\Phi(v, t)$ denotes the Gaussian process with mean

$$
E_{\underline{m}}(\Phi(v, t))=\sum_{j=1}^{n} m_{j} l_{j}(v), \quad \underline{m}=\left(m_{1}, \cdots, m_{n}\right)
$$

and covariance operator $\hat{C}$, then 


$$
E(\cdot)=\frac{1}{(2 \pi)^{n / 2}} \int_{-\infty}^{\infty} d m_{1} \cdots d m_{n} e^{-1 / 2\left(m_{1}^{2}+\cdots m_{n}^{2}\right)} E_{\underline{m}}(\cdot)
$$

is the expectation of the Gaussian process $\Phi(v, t)$ with mean zero and covariance operator

$$
C=\widehat{C}+\sum_{j=1}^{n} l_{j}^{*} l_{j} .
$$

In this way if $\Gamma(0)$ has finite range the process can be decomposed into processes such that $\Gamma(0)=0$. In general, $\Gamma(0)$ can be approximated by operators with finite range, and this shows that the general process is in the closed convex hull of processes with $\Gamma(0)=0$.

REMARK 3.11. In the nonperiodic $(\beta=\infty)$ case (see $\S 5$ ) the decomposition in Remark 3.10 is the ergodic decomposition of the given process into extremal stationary processes. To see this, note that if $\tau_{x}$ denotes translation by $x,\left(\tau_{x} X\right)(t)=X(t+x)$, then

$$
\begin{aligned}
E\left(e^{i X(f)} \tau_{x} e^{i X(g)}\right) & =E\left(e^{i X\left(f+\tau_{x} g\right)}\right)=e^{i m \int^{d t(f+g)} e^{-1 / 2 E\left(X\left(f+\tau_{x} g\right)^{2}\right)}} \\
& =e^{i m \int^{d t f}} e^{-1 / 2 E\left(X(f)^{2}\right)} e^{i m \int^{d t g}} e^{-1 / 2 E(X(g) 2)} e^{-E\left(X(f) X\left(\tau_{x} g\right)\right)} \\
& =E\left(e^{i X(f)}\right) E\left(e^{i X(g)}\right) e^{-E\left(X(f) X\left(\tau_{x} g\right)\right)} .
\end{aligned}
$$

Thus if $\nu_{+}(0)=0, E\left(X(f) X\left(\tau_{x} g\right)\right) \underset{|x| \rightarrow \infty}{\longrightarrow} 0$ and so

$$
E\left(e^{i X(f)} \tau_{x} e^{i X(g)}\right) \underset{|x| \rightarrow \infty}{\longrightarrow} E\left(e^{i X(f)}\right) E\left(e^{i X(g)}\right) .
$$

This asymptotic independence property (clustering) implies that the $\sigma$-algebra of translation invariant functions is trivial and thus the process is ergodic. A similar discussion holds for processes $\Phi(v, t)$ indexed by $V \times \boldsymbol{R}$.

4. The two-sided Markov property on the circle. For periodic processes the usual Markov property does not make sense as the "future" winds back into the "past". The appropriate condition is the two-sided Markov property on the circle. This can be formulated as follows. A periodic linear stochastic process $\Phi(v, t)$ with period $\beta$ can be considered as indexed by $V \times S_{\beta}$. For $I \subset S_{\beta}$, we will denote by $\sum_{I}$ the $\sigma$-algebra generated by $\{\Phi(v, t) \mid v \in V, t \in I\}$, and by $E_{I}$ the corresponding conditional expectation. If $r, s \in S_{\beta}$, we will write $[r, s]$ for the closed interval from $r$ to $s$ in the counterclockwise direction. Thus $[s, r]$ is the closure of $S_{\beta} \backslash[r, s]$. We will say that $\Phi(v, t)$ has the two-sided Markov property on $S_{\beta}$ if and only if for all $r, s \in S_{\beta}, E_{[s, r]} E_{[r, s]}=E_{\{r, s\}} E_{[r, s]}$ where $\{r, s\}=\{s, r\}$ denotes the set consisting of the two elements $r$ and $s$. Equiva- 
lently, it says that $\sum_{[r, s]}$ and $\sum_{[s, r]}$ are independent given $\sum_{\{r, s\}}$.

If $\Phi(v, t)$ is a periodic symmetric process with period $\beta$, then the two-sided Markov property on $S_{\beta}$ implies OS-positivity on $S_{\beta}$. For let $F$ be a bounded measurable function on $R^{n}$, and let $t_{1}, \cdots$, $t_{n} \in[0, \beta / 2], v_{1}, \cdots, v_{n} \in V$. Then $F\left(\Phi\left(v_{1}, t_{1}\right), \cdots, \Phi\left(v_{n}, t_{n}\right)\right), F\left(\Phi\left(v_{1},-t_{1}\right)\right.$, $\left.\cdots, \Phi\left(v_{n},-t_{n}\right)\right)$, are measurable with respect to $\sum_{[0, \beta / 2]}, \sum_{[-\beta / 2,0]}$, respectively, and by symmetry

$$
E_{\{0, \beta / 2\}}\left(F\left(\Phi\left(v_{1}, t_{1}\right), \cdots, \Phi\left(v_{n}, t_{n}\right)\right)\right)=E_{\{0, \beta / 2\}}\left(F\left(\Phi\left(v_{1},-t_{1}\right), \cdots, \Phi\left(v_{n},-t_{n}\right)\right)\right),
$$

hence it follows from the two-sided Markov property on $S_{\beta}$ that

$$
\begin{gathered}
E\left(\bar{F}\left(\Phi\left(v_{1},-t_{1}\right), \cdots, \Phi\left(v_{n},-t_{n}\right)\right) F\left(\Phi\left(v_{1}, t_{1}\right), \cdots, \Phi\left(v_{n}, t_{n}\right)\right)\right) \\
\quad=E\left(\left|E_{\{0, \beta / 2\}}\left(F\left(\Phi\left(v_{1}, t_{1}\right), \cdots, \Phi\left(v_{n}, t_{n}\right)\right)\right)\right|^{2}\right) \geqq 0 .
\end{gathered}
$$

Let us now consider Gaussian process. For such it suffices to look at their second-order properties. So let $\Phi(v, t)$ be a periodic Gaussian linear stochastic process with period $\beta$ that we will consider indexed by $V \times S_{\beta}$. For $I \subset S_{\beta}$ we will denote by $\sigma_{I}$ the closed complex Hilbert space spanned by $\{\Phi(v, t) \mid v \in V, t \in I\}$ in the $L^{2}$-norm, $e_{I}$ will denote the orthogonal projection of $\sigma_{S_{\beta}}$ onto $\sigma_{I}$. We will say that $\Phi(v, t)$ has the two-sided pre-Markov property on $S_{\beta}$ if and only if for all $r, s \in S_{\beta}, e_{[s, r]} e_{[r, s]}=e_{(r, s)} e_{[r, s]}$. For Gaussian processes the two-sided pre-Markov property is equivalent to the two-sided Markov property (see the discussion in Klein [8, proof of Proposition 3.1]).

We now restrict our attention to periodic Gaussian OS-positive processes. We can determine which such processes satisfy the twosided Markov property on the circle.

THEOREM 4.1. Let $\Phi(v, t)$ be a periodic Gaussian OS-positive process with period $\beta$ indexed by $V_{0} \times \boldsymbol{R}$, where $V_{0}$ is a real vector space. Then $\Phi(v, t)$ has the two-sided Markov property on $S_{\beta}$ if and only if $V_{0}$ can be given an inner product with respect to which the covariance function $r: \boldsymbol{R} \rightarrow \mathscr{L}(V)$ of the process can be defined, where $V$ is the completion of $V_{0}$ with this inner product, and such that there exists a nonnegative self-adjoint operator $H$ on $V$ for which $r(t)=e^{-t H}+e^{-(\beta-t) H}$ for $t \in[0, \beta]$.

In particular, given a real Hilbert space $V$ and a nonnegative self-adjoint operator $H$ on $V$, the periodic function $r_{\beta}: \boldsymbol{R} \rightarrow \mathscr{L}(V)$ with period $\beta$ defined by $r_{\beta}(t)=e^{-t H}+e^{-(\beta-t) H}$ for $t \in[0, \beta]$ is the covariance function of a periodic Gaussian OS-positive process with period $\beta$ satisfying the two-sided Markov property on $S_{\beta}$ for any $\beta>0$.

REMARK 4.2. In terms of $V, W, H, j$ as in Theorem 3.8, Theorem 
4.1 says that $\Phi(v, t)$ has the two-sided Markov property on $S_{\beta}$ if and only if $\overline{j V}=W$, i.e., $e^{-t H}$ leaves $\overline{j V}$ invariant for all $t \geqq 0$.

Proof of Theorem 4.1. For a periodic Gaussian process $\Phi(v, t)$ with period $\beta$ the two-sided Markov property on $S_{\beta}$ is equivalent to

$$
e_{[s, r]} e_{[r, s]}=e_{\{r, s\}} e_{[r, s]} \text { for all } r, s \in S_{\beta}
$$

As $e_{[s, r]} e_{[r, s]}=e_{\{r, s\}} e_{[r, s]} \Leftrightarrow e_{[r, s]} e_{[s, r]}=e_{\{s, r\}} e_{[s, r]}$, it suffices to consider $r, s \in S_{\beta}$ with $|r-s| \leqq \beta / 2$. If the process is stationary there is no loss of generality in assuming that $r=0, s=T$ with $0<T \leqq \beta / 2$. It follows that for a periodic Gaussian stationary linear stochastic process $\Phi(v, t)$ with period $\beta$, indexed by $V_{0} \times \boldsymbol{R}$, the two-sided Markov property on $S_{\beta}$ is equivalent to $E(\Phi(u, s) \Phi(v, t))=E(\Phi(u, s) \times$ $\left.\left[e_{\{0, T\}} \Phi(v, t)\right]\right)$ for all $u, v \in V_{0}, t \in[0, T], s \in[T, \beta]$.

Let us now assume that $V$ is a real Hilbert space with inner product $\langle\cdot, \cdot\rangle, H$ a nonnegative self-adjoint operator on $V$, and let us define the periodic function $r: \boldsymbol{R} \rightarrow \mathscr{L}(V)$ with period $\beta$ by $r(t)=e^{-t H}+e^{-(\beta-t) H}$ for $t \in[0, \beta]$. By Theorem $3.8 r(t)$ is the covariance function of a periodic Gaussian OS-positive process $\Phi(v, t)$ with period $\beta$, indexed by $V \times \boldsymbol{R}$. Let us show that $\Phi(v, t)$ satisfy the two-sided Markov property on $S_{\beta}$. To do so we fix $0<T<\beta / 2$, and define $f_{r}(x)=\left(e^{T x}-e^{-T x}\right)^{-1}\left(e^{r x}-e^{-r x}\right)$ for each $0 \leqq r<T$. Then $f_{r}(x)$ is a bounded continuous function on $[0, \infty)$ vanishing at $\infty$. Let $v \in V, t \in[0, T]$, and $v_{1}=f_{T^{-} t}(H) v, v_{2}=f_{t}(H) v$. Then for all $u \in V, s \in[T, \beta]$,

$$
E(\Phi(u, s) \Phi(v, t))=\left\langle u,\left(e^{-(s-t) H}+e^{-(\beta-(s-t)) H}\right) v\right\rangle
$$

On the other hand,

$$
\begin{aligned}
& E\left(\Phi(u, s)\left(\Phi\left(v_{1}, 0\right)+\Phi\left(v_{2}, T\right)\right)\right) \\
& \quad=\left\langle u,\left(e^{-s I I}+e^{-(\beta-s) H}\right) v_{1}+\left(e^{-(s-T) I I}+e^{-(\beta-(8-t)) H}\right) v_{2}\right\rangle .
\end{aligned}
$$

But

$$
e^{-s I I} v_{1}+e^{-(s-T) H} v_{2}=e^{-(s-t) H} v
$$

and

$$
e^{-(\beta-s) H} v_{1}+e^{-(\beta-(s-T)) H} v_{2}=e^{-(\beta-(s-t)) H} v
$$

Thus

$$
E(\Phi(u, s) \Phi(v, t))=E\left(\Phi(u, s)\left[\Phi\left(v_{1}, 0\right)+\Phi\left(v_{2}, T\right)\right]\right)
$$

for all $u \in V, s \in[T, \beta]$, which proves the two-sided Markov property on $S_{\beta}$. 
Let us now prove the converse. So let $\Phi(v, t)$ be a periodic Gaussian OS-positive process with period $\beta$ indexed by $V_{0} \times \boldsymbol{R}$, where $V_{0}$ is a real vector space. By Theorem 3.8 (see also Remark 3.9) $V_{0}$ can be given an inner product $\langle\cdot, \cdot\rangle$ in which it is completed into a real Hilbert space $V$, and there exists a real Hilbert space $W$ having $V$ as a subspace, and a nonnegative self-adjoint operator $H$ on $W$ such that for all $v, u \in V, t, s \in[0, \beta]$,

$$
E(\Phi(u, s) \Phi(v, t))=\left\langle u,\left(e^{-|t-s| H}+e^{-(\beta-|t-s|) H}\right) v\right\rangle .
$$

Moreover $W$ can be taken as $L^{2}\left([0, \infty), V ; d \Gamma_{+}\right)$, where $\Gamma_{+}$is the operator-valued measure on $[0, \infty)$ with values in $\mathscr{L}(V)$ constructed in Theorem 3.3 (see the proof of Theorem 3.8), so $e^{-t H}$ is just multiplication by $e^{-t a}$. So let $\Gamma$ be the operator-valued measure on $\boldsymbol{R}$ with values in $\mathscr{L}(V)$ given by $d \Gamma(a)=d \Gamma_{+}(a)+e^{\beta a} d \Gamma_{+}(-a)$ (see Remark 3.4), and let $\widetilde{W}=L^{2}(\boldsymbol{R}, V ; d \Gamma)$ and $\widetilde{V}$ the subspace of constant functions, i.e., $\widetilde{V}=\{F \in \widetilde{W} \mid$ there exists $v \in V$ such that $F(a)=v$ for all $a \in R\}$. Clearly $\widetilde{V}=V$ as sets, and if $(\cdot, \cdot)$ is the inner product on $\tilde{W},(v, u)=\left\langle v,\left(1+e^{-\beta H}\right) u\right\rangle$, where $\langle\cdot, \cdot\rangle$ is the inner product on $W$. Let $\widetilde{H}$ denote the self-adjoint operator on $\widetilde{W}$ which is the infinitesimal generator of the strongly continuous unitary one-parameter group $U(t)$ on $\widetilde{W}_{c}=L^{2}\left(\boldsymbol{R}, V_{c} ; d \Gamma\right)$ given by $(U(t) F)(a)=e^{i t a} F(a)$ for $F \in \widetilde{W}_{c}$, i.e., such that $U(t)=e^{i t \tilde{H}}$ for all $t \in R$ ( $\widetilde{H}$ is first defined on $\widetilde{W}$ but it leaves $W$ invariant). $\widetilde{H}$ is not bounded from below, but $\widetilde{V}$ is in the domain of $e^{-t \tilde{H}}$ for $t \in[0, \beta / 2]$. Moreover it is not hard to see that the linear span of $\left\{e^{-t \tilde{H}} \widetilde{V} \mid t \epsilon\right.$ $[0, \beta / 2]\}$ is dense in $\widetilde{W}$. Furthermore, for all $u, v \in V, s, t \in[0, \beta / 2]$,

$$
E(\Phi(u,-s) \Phi(v, t))=\left\langle u,\left(e^{-(t+s) H}+e^{-(\beta-(t+s)) H}\right) v\right\rangle=\left(e^{-s \tilde{H}} u, e^{-t \widetilde{H}} v\right) .
$$

We now assume that $\Phi(v, t)$ satisfies the two-sided Markov property on $S_{\beta}$. In particular if $T \in[0, \beta / 2], v \in V$,

$$
E(\Phi(u,-s) \Phi(v, T / 2))=E\left(\Phi(u,-s)\left[e_{\{0, T\}} \Phi(v, T / 2)\right]\right)
$$

for all $u \in V, s \in[0, \beta / 2]$. By an easy computation,

$$
\boldsymbol{e}_{\{0, T\}} \Phi(v, T / 2)=\Phi\left(v_{1}, 0\right)+\Phi\left(v_{1}, T\right),
$$

where

$$
v_{1}=(r(0)+r(T))^{-1} r(T / 2) v
$$

and $r(t)$ is the covariance function of the process defined with respect to the inner product $\langle\cdot, \cdot\rangle$ on $V$ (notice $r(0) \geqq 1, r(T) \geqq 0$ ). Thus

$$
E(\Phi(u,-s) \Phi(v, T / 2))=E\left(\Phi(u,-s)\left[\Phi\left(v_{1}, 0\right)+\Phi\left(v_{1}, T\right)\right]\right) .
$$


Hence

$$
\left(e^{-s \tilde{H}} u, e^{-T / 2 \tilde{H}} v\right)=\left(e^{-s \tilde{H}} u, v_{1}+e^{-T \tilde{H}} v_{1}\right)
$$

for all $u \in V, s \in[0, \beta / 2]$. As the linear $\operatorname{span}$ of $\left\{e^{-s \widetilde{H}} V \mid s \in[0, \beta / 2]\right\}$ is dense in $\tilde{W}$, this implies that $\left(1+e^{-T \widetilde{H}}\right) v_{1}=e^{-T / 2 \tilde{H}} v$, and thus $\left(1+e^{-T \widetilde{H}}\right)^{-1} e^{-T / 2 \widetilde{H}} v=v_{1} \in V$ for all $v \in V$. So let $f(x)=$ $\left(1+e^{-T x}\right)^{-1} e^{-(T / 2) x}$. Then $f(x)$ is a bounded continuous function on $\boldsymbol{R}$ vanishing at $\infty$, and strictly decreasing in $[0, \infty)$. We have proved that $f(\widetilde{H})$ leaves $\widetilde{V}$ invariant. Now we let $E_{+}=\chi_{(0, \infty)}(\widetilde{H})+$ $(1 / 2) \chi_{\{0\}}(\widetilde{H})$ where $\chi_{I}$ is the characteristic function of the set $I$. As $W=L^{2}\left([0, \infty), V ; d \Gamma_{+}\right), \quad \widetilde{W}=L^{2}(\boldsymbol{R}, V ; d \Gamma) \quad$ with $d \Gamma(a)=d \Gamma_{+}(a)+$ $e^{\beta a} d \Gamma_{+}(-a)$, and $V, \widetilde{V}$ are the constant functions in $W, \widetilde{W}$, respectively, it follows that $E_{+} \widetilde{W}=W, E_{+} \widetilde{V}=V, E_{+} \widetilde{H}=H E_{+}$, and thus $E_{+} f(\tilde{H})=f(H) E_{+}$. As $E_{+} \widetilde{V}=V$ and $f(\tilde{H})$ leaves $\widetilde{V}$ invariant, we conclude that $f(H)$ leaves $V$ invariant. But as $f(x)$ is a bounded continuous function on $[0, \infty)$, strictly decreasing and vanishing at $\infty$, it generates a dense sub-algebra of $C_{0}([0, \infty))$ by the StoneWeierstrass theorem. As a consequence $e^{-t H}$ leaves $V$ invariant for all $t \geqq 0$ and thus $V=W$.

REMARK 4.3. The proof of Theorem 4.1 shows that the twosided Markov property for periodic Gaussian OS-positive processes is equivalent to a much weaker property, namely that there exists a $T \in(0, \beta / 2]$ for which

$$
e_{[\beta / 2, \beta]} e_{\{T / 2\}}=e_{\{0, T\}} e_{\{T / 2\}} \text {. }
$$

CoROLlary 4.4. The only periodic Gaussian OS-positive processes indexed by the real line satisfying the two-sided Markov property on the circle are the periodic Ornstein-Uhlenbeck processes (up to multiplication by a constant), i.e., the ones with covariance function of the form $r(t)=C\left(e^{-t a}+e^{-(\beta-t) a}\right)$ for $t \in[0, \beta]$, where $a \geqq 0, C>0$.

Proof. If $r(t)$ is the covariance function of a periodic Gaussian OS-positive process indexed by the real line with period $\beta$, then by Theorem 2.5 there exists a finite measure $\nu_{+}$on $[0, \infty)$ such that $r(t)=\int_{0}^{\infty}\left(e^{-t a}+e^{-(\beta-t) a}\right) d \nu_{+}(a)$ for $t \in[0, \beta]$. In the notation of Theorem 3.8, $V=\boldsymbol{R}, W=L^{2}\left([0, \infty), d \nu_{+}\right), j b$ is the constant function $b$ for all $b \in \boldsymbol{R}$, and $H$ is multiplication by $a$. By Theorem 4.1 (see also Remark 4.2) the process satisfies the two-sided Markov property on $S_{\beta}$ if and only if $V=W$, i.e., if and only if $L^{2}([0, \infty)$, $\left.d \nu_{+}\right)$is one-dimensional, and thus if and only if $\nu_{+}(a)=C \delta\left(a-a_{0}\right)$ 
for some $C>0, a_{0} \geqq 0$, where $\delta(a)$ is the $\delta$-function, (i.e., the probability measure that gives measure one to the set $\{0\})$. This proves the corollary.

The analogous result is true for generalized free Euclidean fields.

COROLLARY 4.5. The only periodic generalized free Euclidean fields which satisfy the two-sided Markov property on the circle are the periodic free Euclidean fields (up to multiplication by a constant).

Proof. As in the proof of Corollary 4.4, all we have to do is to identify $V, W$, and $H$ of Theorem 3.8. The periodic generalized free Euclidean field with period $\beta$ is the periodic Gaussian linear stochastic process $\Phi(f, t)$ indexed by $\mathscr{S}_{r}\left(\boldsymbol{R}^{d}\right) \times R$ with covariance

$$
\begin{gathered}
E(\Phi(f, t) \Phi(g, s))=\int_{0}^{\infty} d \rho\left(m^{2}\right)\left(f,\left[2 \sqrt{-\Delta+m^{2}}\left(1-\exp \left(-\beta \sqrt{-\Delta+m^{2}}\right)\right)\right]^{-1}\right. \\
\left.\quad \times\left[\exp \left(-|t-s| \sqrt{-\Delta+m^{2}}\right)+\exp \left(-(\beta-|t-s|) \sqrt{-\Delta+m^{2}}\right)\right] g\right),
\end{gathered}
$$

for $f, g \in \mathscr{S}_{r}\left(\boldsymbol{R}^{d}\right), t, s \in[0, \beta]$, where $\rho$ is a measure on $[0, \infty)$ with $\int_{1}^{\infty} m^{-1} d \rho\left(m^{2}\right)<\infty$ (and $\int_{0}^{1} \ln m^{-1} d \rho\left(m^{2}\right)<\infty$ if $d=2, \int_{0}^{1} m^{-1} d \rho\left(m^{2}\right)<\infty$ if $d=1$ ), and $(\cdot, \cdot)$ is the $L^{2}$-inner product. Thus $V$ is the completion of $\mathscr{S}_{r}\left(\boldsymbol{R}^{d}\right)$ in the inner product

$$
\langle f, g\rangle=\int_{0}^{\infty} d \rho\left(m^{2}\right)\left(f,\left[2 \sqrt{-\Delta+m^{2}}\left(1-\exp \left(-\beta \sqrt{-\Delta+m^{2}}\right)\right)\right]^{-1} g\right),
$$

$W$ is the completion of $\mathscr{S}_{r}\left(R^{d} \times[0, \infty)\right)$ in the inner product

$$
\begin{aligned}
& \langle F, G\rangle=\int_{0}^{\infty} d \rho\left(m^{2}\right)\left(F\left(\cdot, m^{2}\right),\left[2 \sqrt{-\Delta+m^{2}}\right.\right. \\
& \left.\left.\quad \times\left(1-\exp \left(-\beta \sqrt{-\Delta+m^{2}}\right)\right)\right]^{-1} G\left(\cdot, m^{2}\right)\right), \\
& H=\sqrt{-\Delta+m^{2}}, \text { i.e., }\left(e^{-t I I} F\right)\left(x, m^{2}\right)=\exp \left(-t \sqrt{\left.-\Delta+m^{2}\right)} F\left(\cdot, m^{2}\right)\right)(x),
\end{aligned}
$$

and $(j f)\left(x, m^{2}\right)=f(x)$ for $f \in \mathscr{S}_{r}\left(\boldsymbol{R}^{d}\right)$ so we can identify $V$ and $j V$. By Remark 4.2 the process has the two-sided Markov property on $S_{\beta}$ if and only if $e^{-t H} V \subset V$ for all $t \geqq 0$, hence if and only if $\rho\left(m^{2}\right)$ $C \delta\left(m^{2}-m_{0}^{2}\right)$ for some $m_{0}^{2} \geqq 0, C>0$, i.e., if and only if the process is a multiple of the periodic free Euclidean field with period $\beta$.

In general, given two linear stochastic processes $\Phi(v, t)$ and $\Psi(w, t)$ indexed by $V \times \boldsymbol{R}$ and $W \times \boldsymbol{R}$, respectively, where $V$ and $W$ are real vector spaces, we will say that $\Phi$ is a restriction of $\Psi$, or that $\Psi$ is an extension of $\Phi$, if $V$ can be identified with a subspace 
of $W$ and under this identification $\Phi(v, t)$ and $\Psi(v, t)$ are equivalent processes indexed by $V \times \boldsymbol{R}$. Restrictions of OS-positive processes are always OS-positive, but restrictions of two-sided Markov processes are not necessarily two-sided Markov, they are only OS-positive. In the Gaussian case this is the general situation, i.e., periodic Gaussian OS-positive processes are restrictions of periodic Gaussian two-sided Markov processes (by a periodic Gaussian two-sided Markov process we mean a periodic stationary stochastic linear process, continuous in quadratic mean, satisfying the two-sided Markov property on the circle; in other words, a periodic Gaussian OS-positive process satisfying the two-sided Markov property on the circle).

THEOREM 4.6. Every periodic Gaussian OS-positive process has a periodic Gaussian two-sided Markov extension. Moreover there exists a minimal such extension.

Proof. Let $\Phi(v, t)$ be a periodic Gaussian OS-positive process indexed by $V \times R$ with period $\beta$, and let $W$ and $H$ be as in Theorem 3.8 (we identify $V$ and $j V$ ). Then the periodic Gaussian process $\Psi(w, t)$ with period $\beta$ indexed by $W \times \boldsymbol{R}$ having covariance function $e^{-t H}+e^{-(\beta-t) H}$ for $t \in[0, \beta]$ is an extension of $\Phi(v, t)$ satisfying the two-sided Markov property on $S_{\beta}$ by Theorem 4.1. Moreover $\Psi(w, t)$ is the minimal periodic Gaussian two-sided Markov extension, for suppose $\Psi^{\prime}\left(w^{\prime}, t\right)$ is another such extension indexed by $W_{0}^{\prime} \times R$ where $W_{0}^{\prime}$ is a real vector space. Then by Theorem 4.1 there exists an inner product $\langle\cdot, \cdot\rangle$ on $W_{0}^{\prime}$ and a nonnegative self-adjoint operator $H^{\prime}$ on the real Hilbert space $W^{\prime}$ which is the completion of $W_{0}^{\prime}$, such that for $w_{1}^{\prime}, w_{2}^{\prime} \in W^{\prime}, t \in[0, \beta]$,

$$
E\left(\Psi^{\prime}\left(w_{1}^{\prime}, 0\right) \Psi^{\prime}\left(w_{2}^{\prime}, t\right)\right)=\left\langle w_{1}^{\prime},\left(e^{-t H^{\prime}}+e^{-(\beta-t) H \prime}\right) w_{2}^{\prime}\right\rangle .
$$

In particular, as $\Psi^{\prime}\left(w^{\prime}, t\right)$ is an extension of $\Phi(v, t)$, if $v_{1}, v_{2} \in V$, and $r(t)$ is the covariance function of $\Phi(v, t)$, we have $\left\langle v_{1}, r(t) v_{2}\right\rangle=$ $\left\langle v_{1},\left(e^{-t H^{\prime}}+e^{-(\beta-t) H^{\prime}}\right) v_{2}\right\rangle$ for $t \in[0, \beta]$. So let $W^{\prime \prime}$ be the closed linear span of $\left\{e^{-t H^{\prime}} V \mid t \geqq 0\right\}$, and $H^{\prime \prime}$ the restriction of $H^{\prime}$ to $W^{\prime \prime}$. It follows from the uniqueness in Theorem 3.8 that $W^{\prime \prime}, H^{\prime \prime}$ can be identified with $W H$ and thus $\Psi^{\prime}\left(w^{\prime}, t\right)$ is an extension of $\Psi(w, t)$.

5. The two-sided Markov property for nonperiodic processes. Let $\Phi(v, t)$ be a linear stochastic process indexed by $V \times \boldsymbol{R}$, where $V$ is a real vector space. If $I \subset \boldsymbol{R}$ we will denote by $\Sigma_{I}$ the $\sigma$-algebra generated by $\{\Phi(v, t) \mid v \in V, t \in I\}$, and by $E_{I}$ the corresponding conditional expectation. We say that $\Phi(v, t)$ has the Markov property if and only if $E_{[t, \infty)} E_{(-\infty, t]}=E_{\{t\}} E_{(-\infty, t]}$ for all $t \in R$; and that $\Phi(v, t)$ has the two-sided Markov property if and only if 
$E_{(-\infty, s] \cup[t, \infty)} E_{[s, t]}=E_{\{s, t\}} E_{[s, t]}$ for all $s, t \in R, s \leqq t$.

The Markov property implies the two-sided Markov property. This follows from the fact that the Markov property requires that $E_{[s, t]} E_{(-\infty, s]}=E_{\{s\}} E_{(-\infty, s]}$ and $E_{[s, t]} E_{[t, \infty)}=E_{\{t\}} E_{[t, \infty)}$ for all $s, t \in R$, and that the two-sided Markov property is equivalent to $E_{[s, t]} E_{(-\infty, s] \cup[t, \infty)}=$ $E_{\{s, t\}} E_{(-\infty, s] \cup[t \infty)}$ for all $s, t \in R, s \leqq t$. The converse is however not true. In general the two-sided Markov property does not imply the Markov property. We will prove, though, that for (nonperiodic) Gaussian OS-positive processes the two-sided Markov property and the Markov property are equivalent.

By a (nonperiodic) Gaussian OS-positive process we will mean a Gaussian linear stochastic process indexed by $V \times \boldsymbol{R}$, where $V$ is a real vector space, which is stationary, continuous is quadratic mean, and satisfies Osterwalder-Schrader positivity (see Klein [8]). Such a process is automatically symmetric. Gaussian OS-positive processes were studied by Klein [8]. The results we will need are listed in the next theorem.

TheOREM 5.1. (Klein [8]). Let $\Phi(v, t)$ be a Gaussian OS-positive process indexed by $V_{0} \times \boldsymbol{R}$, where $V_{0}$ is a real vector space. Then there exists a real Hilbert space $W_{0}$ such that $V_{0}$ can be identified with a subspace of $W_{0}$, and a nonnegative self-adjoint operator $H_{0}$ on $W_{0}$ such that for all $u, v \in V_{0}, t, s \in R$,

$$
E(\Phi(u, s) \Phi(v, t))=\left\langle u, e^{-|t-s| H_{0}} v\right\rangle .
$$

Conversely, given such $a W_{0}$ and $H_{0}$, there exists a Gaussian OSpositive process $\Phi(v, t)$ indexed by $V_{0} \times \boldsymbol{R}$ such that $\left(^{*}\right)$ holds. Moreover, $W_{0}$ and $H_{0}$ are unique (up to isomorphisms) if we require that the linear span of $\left\{e^{-t H_{0}} V_{0} \mid t \geqq 0\right\}$ be dense in $W_{0}$.

Furthermore, $\Phi(v, t)$ is a Markov process if and only if $V_{0}$ is dense in $W_{0}$.

CoRollary 5.2. Let $\Phi(v, t)$ be a Gaussian OS-positive process indexed by $V_{0} \times \boldsymbol{R}$, where $V_{0}$ is a real vector space. Then there exists a real Hilbert space $W$ such that $V_{0}$ can be identified with a subspace of $W$, and a nonnegative self-adjoint operator $H$ on $W$ with spectral projection $E$, such that for all $u, v \in V_{0}, t, s \in \boldsymbol{R}$,

$$
E(\Phi(u, s) \Phi(v, t))=\left\langle u,\left(e^{-|t-s| I I}+E(\{0\})\right) v\right\rangle .
$$

Conversely, given such a $W$ and $H$, there exists a Gaussian OSpositive process $\Phi(v, t)$ indexed by $V_{0} \times \boldsymbol{R}$ such that (**) holds. Moreover $W$ and $H$ are unique if we require that the linear span of $\left\{e^{-t H} V_{0} \mid t \geqq 0\right\}$ be dense in $W$. 
Proof. Due to Theorem 5.1, it suffices to show that given a real vector space $V_{0}$, then if $W_{0}$ is a real Hilbert space having $V_{0}$ as a subspace, and $H_{0}$ is a nonnegative self-adjoint operator on $W_{0}$, then there exists a real Hilbert space $W$ with $V_{0}$ a subspace of $W$, and a nonnegative self-adjoint operator $H$ on $W$ with spectral protection $E$, such that for all $u, v \in V_{0}$, and $t \geqq 0$,

$$
\left\langle u, e^{-t H_{0}} v\right\rangle_{0}=\left\langle u,\left(e^{-t H}+E(\{0\})\right) v\right\rangle,
$$

where $\langle\cdot, \cdot\rangle_{0},\langle\cdot, \cdot\rangle$ are the inner products in $W_{0}, W$, respectively; and vice-versa.

To do so let $E_{0}$ be the spectral projection of $H_{0}$, and introduce a new inner product $\langle\cdot, \cdot\rangle$ on $W_{0}$ by

$$
\left\langle w_{1}, w_{2}\right\rangle=(1 / 2)\left\langle w_{1}, E_{0}(\{0\}) w_{2}\right\rangle_{0}+\left\langle w_{1},\left(1-E_{0}(\{0\})\right) w_{2}\right\rangle_{0}
$$

for all $w_{1}, w_{2} \in W_{0}$. The new inner product is equivalent to the original one and thus $W_{0}$ with the new inner product form a real Hilbert space $W$ with $V_{0} \subset W$. Let $J: W_{0} \rightarrow W$ be defined by $J w=w$ for all $w \in W_{0}$. Then $J$ is a real vector space isomorphism, bounded with bounded inverse, and $J V_{0}=V_{0}$. Let $H=J H_{0} J^{-1}$. Then $H$ is a nonnegative self-adjoint operator on $W$ such that if $E$ is its spectral projection, $E(\{0\})=J E_{0}(\{0\}) J^{-1}$, and for all $u, v \in V_{1}$ and $t \geqq 0$

$$
\left\langle u,\left(e^{-t H}+E(\{0\})\right) v\right\rangle=\left\langle u, J\left(e^{-t H_{0}}+E_{0}(\{0\})\right) J^{-1} v\right\rangle=\left\langle u, e^{-t H_{0}} v\right\rangle_{0} .
$$

The converse is proved in a similar way.

We now give a characterization of Gaussian OS-positive processes satisfying the two-sided Markov property.

THEOREM 5.3. Let $\Phi(v, t)$ be a Gaussian OS-positive process indexed by $V_{0} \times \boldsymbol{R}$, where $V_{0}$ is a real vector space, and let $W, H, E$ be as in Corollary 5.2. Then $\Phi(v, t)$ has the two-sided Markov property if and only if $V_{0}$ is dense in $W$.

Proof. The proof of Theorem 4.1 applies with the obvious modifications. The fact that if $V_{0}$ is dense in $W$ then $\Phi(v, t)$ satisfies the two-sided Markov property also follows from the fact that if $V_{0}$ is dense in $W$ then $V_{0}$ is dense in $W_{0}$ (of Theorem 5.1) by the construction in the proof of Corollary 5.2, and thus by Theorem 5.1 $\Phi(v, t)$ satisfies the Markov property, which implies the two-sided Markov property.

COROLlaRY 5.4. Let $\Phi(v, t)$ be a Gaussian OS-positive process indexed by $V_{0} \times \boldsymbol{R}$, where $V_{0}$ is a real vector space. Then $\Phi(v, t)$ 
has the Markov property if and only if it has the two-sided Markov property.

Proof. It follows easily from Theorem 5.1, Corollary 5.2 (and its proof), and Theorem 5.3.

We can now relate the two-sided Markov property on the circle for periodic Gaussian OS-positive processes to the Markov property for the (nonperiodic) Gaussian OS-positive process obtained by letting the period $\beta$ go to $\infty$ (see Remark 3.6). More precisely, we have:

THEOREM 5.5. Let $\Phi_{\beta_{0}}(v, t)$ the periodic Gaussian OS-positive process with period $\beta_{0}$ indexed by $V_{0} \times \boldsymbol{R}$, where $V_{0}$ is a real vector space, and for each $\beta>0$ let $\Phi_{\beta}(v, t)$ be the periodic OS-positive process with period $\beta$ indexed by $V_{0} \times \boldsymbol{R}$ constructed from $\Phi_{\beta}(v, t)$ in Remark 3.5, and let $\Phi_{\infty}(v, t)$ be the (nonperiodic) Gaussian OS-positive process obtained by letting $\beta \rightarrow \infty$ (Remark 3.6). Then the following are equivalent:

(i) $\Phi_{\beta_{0}}(v, t)$ has the two-sided Markov property on $S_{\beta_{0}}$.

(ii) $\Phi_{\beta}(v, t)$ has the two-sided Markov property on $S_{\beta}$ for all $\beta>0$.

(iii) $\Phi_{\infty}(v, t)$ has the Markov property.

Proof. It follows from Theorems 4.1, 5.3, and Corollary 5.4. $\square$

\section{REFERENCES}

1. S. Albeverio and R. Hoegh-Krohn, Homogeneous random fields and statistical mecha. nics, J. Functional Analysis, 19 (1975), 242-272.

2. W. Driessler, L. Landau and J. Perez, Estimates of critical lengths and critical temperatures for classical and quantum lattice systems, J. Stat. Physics, 20 (1979), 123-162.

3. R. Hoegh-Krohn, Relativistic quantum statistical mechanics in two-dimensional spacetime, Comm. Math. Phys., 38 (1974), 195-224.

4. A. Klein, A characterization of Osterwalder-Schrader path spaces by the associated semigroup, Bull. Amer. Math. Soc., 82 (1976), 762-764.

5. - When do Euclidean fields exist?, Let. Math. Phys., 1 (1976), 131-133.

6. The semigroup characterization of Osterwalder-Schrader path spaces and the construction of Euclidean fields, J. Functional Analysis, 27 (1978), 277-291.

7. - A generalization of Markov processes, Ann. Probability, 6 (1978), 128-132.

8. - Gaussian OS-Positive Processes, Z. Wahrscheinlichkeitstheorie verw. Ge. biete, 40 (1977), 115-124.

9. A. Klein and L. Landau, Stochastic processes associated with KMS states, J. Functional Analysis, to appear.

10. M. Krein, General Theorems About Positive Definite Functionals, in "Some Questions in The Theory of Moments" by N. Aheizer and M. Krein, American Mathematical Society, Providence, Rhode Island, 1962, 124-153.

11. E. Nelson, Construction of quantum fields from Markoff fields, J. Functional Analy. 
sis, 12 (1973), 97-112.

12. E. Neison, The free Markoff field, J. Functional Analysis, 12 (1973), 211-227.

13. K. Osterwalder and R. Schrader, Axioms for Euclidean Green's functions, $I$, Comm. Math. Phys., 31 (1973), 83-112; II, Comm. Math. Phys., 42 (1975), 281-305.

14. B. Simon, The $P(\varphi)_{2}$ Euclidean (Quantum) Field Theory, Princeton Series in Physics, Princeton University Press, Princeton, N.J., 1974.

Received November 29, 1979. The first author was partially supported by the N.S.F. under grant MCS 78-01433.

University of California

IRVINE, CA 92717

AND

Bedford College

UNIVERSITY OF LONDON

LONDON NWI 4NS

ENGLAND 



\section{PACIFIC JOURNAL OF MATHEMATICS}

\section{EDITORS}

DONALD BABBITT (Managing Editor)

University of California

Los Angeles, CA 90024

Hugo RossI

University of Utah

Salt Lake City, UT 84112

C. C. MOORE and ANDREW OGG

University of California

Berkeley, CA 94720
J. DugundjI

Department of Mathematics

University of Southern California

Los Angeles, CA 90007

R. FINN and J. MILGRAM

Stanford University

Stanford, CA 94305

\section{ASSOCIATE EDITORS}
R. ARENS
E. F. BECKENBACH
B. H. NeUmanN
F. WOLF
K. YosHIDA

\section{SUPPORTING INSTITUTIONS}

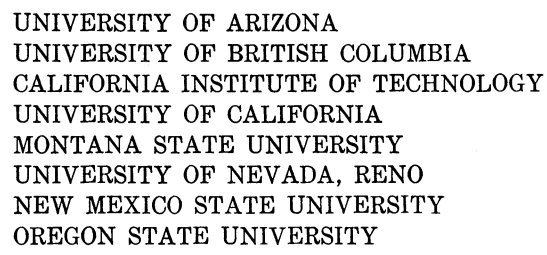

UNIVERSITY OF ARIZONA

UNIVERSITY OF BRITISH COLUMBIA CALIFORNIA INSTITUTE OF TECHNOLOGY

UNIVERSITY OF CALIFORNIA

MONTANA STATE UNIVERSITY

UNIVERSITY OF NEVADA, RENO

NEW MEXICO STATE UNIVERSITY OREGON STATE UNIVERSITY

\author{
UNIVERSITY OF OREGON \\ UNIVERSITY OF SOUTHERN CALIFORNIA \\ STANFORD UNIVERSITY \\ UNIVERSITY OF HAWAII \\ UNIVERSITY OF TOKYO \\ UNIVERSITY OF UTAH \\ WASHINGTON STATE UNIVERSITY \\ UNIVERSITY OF WASHINGTON
}

The Supporting Institutions listed above contribute to the cost of publication of this Journal, but they are not owners or publishers and have no responsibility for its content or policies.

Mathematical papers intended for publication in the Pacific Journal of Mathematics should be in typed form or offset-reproduced, (not dittoed), double spaced with large margins. Please do not use built up fractions in the text of the manuscript. However, you may use them in the displayed equations. Underline Greek letters in red, German in green, and script in blue. The first paragraph or two must be capable of being used separately as a synopsis of the entire paper. Please propose a heading for the odd numbered pages of less than 35 characters. Manuscripts, in triplicate, may be sent to any one of the editors. Please classify according to the scheme of Math. Reviews, Index to Vol. 39. Supply name and address of author to whom proofs should be sent. All other communications should be addressed to the managing editor, or Elaine Barth, University of California, Los Angeles, California, 90024.

50 reprints to each author are provided free for each article, only if page charges have been substantially paid. Additional copies may be obtained at cost in multiples of 50 .

The Pacific Journal of Mathematics is issued monthly as of January 1966. Regular subscription rate: $\$ 102.00$ a year (6 Vols., 12 issues). Special rate: $\$ 51.00$ a year to individual members of supporting institutions.

Subscriptions, orders for numbers issued in the last three calendar years, and changes of address shoud be sent to Pacific Journal of Mathematics, P.O. Box 969, Carmel Valley, CA 93924, U.S.A. Old back numbers obtainable from Kraus Per!odicals Co., Route 100, Millwood, NY 10546.

\section{PUBLISHED BY PACIFIC JOURNAL OF MATHEMATICS, A NON-PROFIT CORPORATION}

Printed at Kokusai Bunken Insatsusha (International Academic Printing Co., Ltd.). 8-8, 3-chome, Takadanobaba, Shinjuku-ku, Tokyo 160, Japan.

Copyright (C) 1981 by Pacific Jounal of Mathematics Manufactured and first issued in Japan 


\section{Pacific Journal of Mathematics}

Vol. 94, No. $2 \quad$ June, 1981

Thomas E. Armstrong and William David Sudderth, Nearly strategic

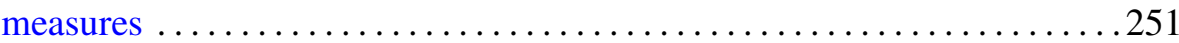

John J. Buoni, Artatrana Dash and Bhushan L. Wadhwa, Joint Browder

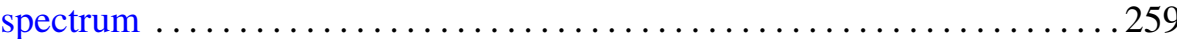

Jack Paul Diamond, Hypergeometric series with a $p$-adic variable . .......265

Raymond Frank Dickman, Jack Ray Porter and Leonard Rubin,

Completely regular absolutes and projective objects ............ 277

James Kenneth Finch, On the local spectrum and the adjoint ........... 297

Benno Fuchssteiner, An abstract disintegration theorem ............ 303

Leon Gerber, The volume cut off a simplex by a half-space $\ldots \ldots \ldots \ldots 311$

Irving Leonard Glicksberg, An application of Wermer's subharmonicity

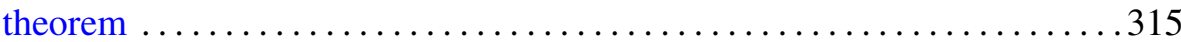

William Goldman, Two examples of affine manifolds ............... 327

Yukio Hirashita, On the Weierstrass points on open Riemann surfaces .....331

Darrell Conley Kent, A note on regular Cauchy spaces ............. 333

Abel Klein and Lawrence J. Landau, Periodic Gaussian

Osterwalder-Schrader positive processes and the two-sided Markov

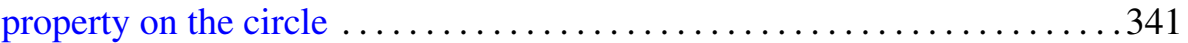

Brenda MacGibbon, $\mathscr{K}$-Borelian embeddings and images of Hausdorff

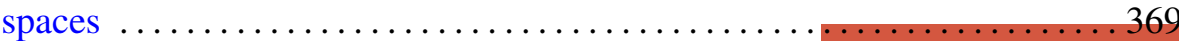

John R. Myers, Homology 3-spheres which admit no PL involutions . . . . . 379

Boon-Hua Ong, Invariant subspace lattices for a class of operators . . . . . 385

Chull Park, Representations of Gaussian processes by Wiener processes . . . 407

Lesley Millman Sibner and Robert Jules Sibner, A sub-elliptic estimate

for a class of invariantly defined elliptic systems $\ldots \ldots \ldots \ldots \ldots \ldots .417$

Justin R. Smith, Complements of codimension-two submanifolds. III.

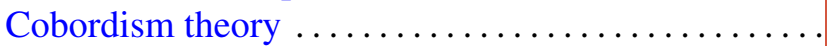

William Albert Roderick Weiss, Small Dowker spaces

David J. Winter, Cartan subalgebras of a Lie algebra and its ideals. II ... 